\title{
Finite time boundedness of neutral high-order Hopfield neural networks with time delay in the leakage term and mixed time delays
}

\author{
Chaouki Aouiti ${ }^{\mathrm{a}}$, Patrick Coirault ${ }^{\mathrm{b}}$, Foued Miaadi ${ }^{\mathrm{a}}$, Emmanuel Moulay ${ }^{\mathrm{c}}$ \\ ${ }^{a}$ University of Carthage, Faculty of Sciences of Bizerta, Department of Mathematics, Research Units of Mathematics and Applications UR13ES47, 7021 Zarzouna, \\ Bizerta, Tunisia \\ ${ }^{b}$ LIAS (EA 6315), University of Poitiers, 2 rue Pierre Brousse, 86073 Poitiers Cedex 9, France \\ ${ }^{c} X$ LIM (UMR CNRS 7252), University of Poitiers, 11 bd Marie et Pierre Curie, 86962 Futuroscope Chasseneuil Cedex, France
}

\begin{abstract}
This article deals with the finite time boundedness (FTB) and FTB-stabilization problem for a general class of neutral high-order Hopfield neural networks (NHOHNNs) with time delay in the leakage term and mixed time delays. The mixed time delays consist of both discrete time-varying delays and infinite distributed delays. By using the topological degree theory, sufficient conditions are established to prove the existence of equilibrium points. Then, the Lyapunov-Krasovskii functional (LKF) method is used to prove sufficient conditions for the FTB. These conditions are in the form of linear matrix inequalities (LMIs) and can be numerically checked. Furthermore, a state feedback control is constructed to solve the FTB-stabilization problem. Finally, some numerical examples are presented to show the effectiveness of our main results.
\end{abstract}

Keywords: High-order neural networks, finite time boundedness, topological degree, stabilization, Lyapunov-Krasovskii functional, LMI, neutral systems, leakage delay .

\section{Introduction}

Neural Networks (NNs) have been widely studied due to their practical applications in lots of areas such as model identification, signal processing, image processing, pattern recognition, optimization problems, associative memories [1, 2, 3, 4]. The majority of these applications requires the stability of the designed NNs. It should be pointed out that the delay has a great effect on the system performances. Therefore, the stability analysis of delayed NNs has attracted the attention of many researchers and a lot of results have been obtained (see for instance [5, 6, 7, 8, 9, 10, 11]). In [10, 12], the authors discussed the case of constant delays and in [6, 9, 11, 13] the authors analyzed the stability of NNs with continuously distributed delays.

Recently, another kind of delay, variously known as leakage delay, is investigated in [14, 15, 16, 17]. It has been proved that this kind of delay tends to render the systems of NNs unstable. The effect of the leakage delay on stability is one of the important research topics in the field of the stability of NNs [18]. Many researchers analyzed the effect of the leakage delay on the Lyapunov stability of various kinds of NNs such that bidirectional associative memory NNs [19, 20] or impulsive NNs [21, 22]. However, it should be pointed out that manipulating this kind of delay is not easy. In addition, a kind of time delay

\footnotetext{
${ }^{*}$ Corresponding author

Email address: patrick.coirault@univ-poitiers.fr (Patrick Coirault)

URL: chaouki.aouiti@fsb.rnu.tn (Chaouki Aouiti), foued.miaadi@yahoo.com (Foued Miaadi), emmanuel.moulay@univ-poitiers.fr (Emmanuel Moulay)
}

systems, appointed neutral-type delay systems, is used by many authors due to their practical applications [23]. There are several results discussing the stability of neutral-type NNs, see for instance [24, 25, 26] and references therein.

In order to render the stability criteria less conservative with respect to the delays, several methods are developed in the literature [27, 28, 29, 30, 31, 32, 33, 34]. Most of these methods are based on a Lyapunov functional and associated LMIs because these inequalities can be numerically checked. However, it is well known that when a novel Lyapunov functional is designed for reducing the conservatism with respect to the delays a greater complexity in terms of inequalities and variables to be calculated can appear. Therefore, the problem of reducing simultaneously the number of decision variables and the conservatism with respect to the delays arises.

Most of the previous works on stability were mainly based on the classical Lyapunov stability which is associated with an infinite time interval. However, only a finite time interval is considered in practical applications [35]. In 1953, Kamenkov has introduced in [36] the concept of finite time boundedness (FTB). Dorato reported in [37] that FTB and Lyapunov stability are two independent concepts. Many studies have addressed the FTB problem of NNs, see for instance [38, 39, 40, 41, 42, 43, 44, 45, 46, 47, 48, 49]. For neutral-type NNs, the FTB was studied in [42, 47]. In [43, 49] and [41], the class of uncertain NNs with Markovian jumps and the complex-valued NNs are studied respectively. Also the impulsive NNs is investigated in [39] and the authors of [40, 38] deal with the problem of FTB for Memristive NNs and TS-fuzzy system with time-varying delay respectively. Moreover, all the previous results on FTB 
are for lower order NNs. This class of lower order NNs has several limitations (see for instance [50, 51]) which leads to consider the class of NNs with high-order connections. This class of high-order NNs has stronger approximation characteristics, important storage capacity, faster convergence rate and higher fault tolerance than lower-order NNs [52]. To the best of the authors' knowledge, the FTB and FTB-stabilization of neutral high-order Hopfield NNs (NHOHNNs) with a leakage delay and with both discrete and distributed delays, called mixed time delays, have not been fully investigated in the literature. Here is the main goal of our article. The delays considered in our article contain an infinite distributed delay which occurs in practice [23]. Indeed, there is no result for the FTB of NNs with infinite distributed delays. Despite important studies available yet to deal with the FTB concept [42, 43, 47, 48, 49], there is no result for the FTB of NNs with non-differentiable time-varying delay. Here is also a novelty of our article.

In our article, the FTB and FTB-stabilization are analyzed for a general class of NHOHNNs with time delay in the leakage term and mixed time delays. The main contributions are as follows:

(i) the LKF and LMIs techniques are used to study the effect of the leakage delay on the FTB results of the concerned NHOHNNs;

(ii) simplified LMI conditions are established to reduce the conservatism with respect to the delays and provide a less computational load simultaneously;

(iii) by using the FTB analysis, some conditions are given for the FTB-stabilization of the concerned NHOHNNs with a leakage delay. Moreover, the NHOHNNs considered in this article are subjected to non-differentiable timevarying delays.

The article is organized as follows. In Section 2, some preliminaries useful for the study of the class of NHOHNNs are presented. The existence of equilibrium points is addressed in Section 3. Then, the FTB and the FTB-stabilization are studied in Section 4 Numerical examples are presented in Section 5 to illuminate the validity of the proposed results. Finally, some concluding remarks are drawn in Section 6 .

\section{Preliminaries}

In this article, we use the following notations:

- $\mathbb{R}, \mathbb{Z}^{+}, \mathbb{R}^{n}$ and $\mathbb{R}^{n \times n}$ stand for the set of real numbers, the set of positive integers, the $n$-dimensional real space equipped with the euclidean norm $\|$.$\| and the set of n \times n$ real matrices respectively;

- $A^{T}, A^{-1}, \quad A>0, \quad A<0$ and $I$ means respectively the transpose of $A$, the inverse of $A$, the matrix $A$ is positive definite, the matrix $-A$ is positive definite and the identity matrix with appropriate dimensions;
- $\lambda_{\max }(A), \quad \lambda_{\min }(A)$ and $*$ stand for the maximum eigenvalue of $A$, the minimum eigenvalue of $A$ and the symmetric block in one symmetric matrix respectively;

- $X^{+}=\left(\left|x_{i j}\right|\right)_{n \times n}$ where $X=\left(x_{i j}\right)_{n \times n}$;

- for any interval $I \subset \mathbb{R}$ and $V \subset \mathbb{R}^{k}(1 \leq k \leq n), C(I, V)$ and $C_{b}^{1}(I, V)$ stand respectively for the set $\{\phi: I \rightarrow V: \phi$ is continuous $\}$ and $\{\phi: I \rightarrow V: \phi$ is continuously differentiable and bounded $\}$.

We consider the following neutral high-order Hopfield neural networks (NHOHNNs) with time delay in the leakage term and mixed time delays

$$
\left\{\begin{aligned}
\dot{x}_{i}(t) & =-c_{i} x_{i}(t-\sigma)+\sum_{j=1}^{n} a_{i j} f_{j}\left(x_{j}(t-\tau(t))\right) \\
& +\sum_{j=1}^{n} \sum_{k=1}^{n} T_{i j k} f_{k}\left(x_{k}(t-\tau(t))\right) f_{j}\left(x_{j}(t-\tau(t))\right) \\
& +\sum_{j=1}^{n} b_{i j} \int_{-\infty}^{t} k_{j}(t-s) f_{j}\left(x_{j}(s)\right) d s \\
& +\sum_{j=1}^{n} d_{i j} \dot{x}_{j}(t-h(t))+J_{i}, \quad t>0, i=1, \ldots, n \\
x(s) \quad & \phi(s), \quad s \in(-\infty, 0]
\end{aligned}\right.
$$

where $c_{i}>0, C=\operatorname{diag}\left(c_{1}, \ldots, c_{n}\right), a_{i j}, b_{i j}$ and $d_{i j}$ are the interconnection weight coefficients of the neurons, $T_{i j k}$ the secondorder synaptic weights of the NNs, $J_{i}$ an external input vector, $x(t)=\left(x_{1}(t), \ldots, x_{n}(t)\right)^{T}$ the neuron state vector of the NNs, $\dot{x}$ the time derivative of the neuron state,

$$
f(x(.))=\left(f_{1}\left(x_{1}(.)\right), \ldots, f_{n}\left(x_{n}(.)\right)\right)^{T}
$$

the neuron activation function such that $f_{i}(0)=0$ for all $1 \leq i \leq$ $n, \sigma \geq 0$ a constant which is the leakage delay,

$$
K=\operatorname{diag}\left(k_{1}(.), \ldots, k_{n}(.)\right)
$$

the delay kernel, $\tau($.$) and h($.$) the time-varying transmission$ delays satisfying $0 \leq \tau(t) \leq \tau, 0 \leq h(t) \leq \bar{h}$ and $\dot{h}(t) \leq h^{*}<1$. The initial condition satisfies $\phi(.) \in C_{b}^{1}\left((-\infty, 0], \mathbb{R}^{n}\right)$ where the norm is defined by

$$
\|\phi\|_{\bar{h}}=\max \left\{\sup _{s \leq 0}\|\phi(s)\|, \sup _{-\bar{h} \leq s \leq 0}\|\dot{\phi}(s)\|\right\} .
$$

The term of the right-hand side of System (1) involving the time derivative of the state renders the system of neutral-type (see for instance [53]). Several studies have been done on System (1), especially in [8], where the global exponential stability is treated for $\sigma=0$ and with Markovian jump parameters. In [54], the authors studied the stability of the almost automorphic solutions for the same model but without taking into consideration infinite distributed delays. Note that such System (1) contains many of the well-known models as special cases.

Let us introduce the following three assumptions:

(H1) for all $x, y \in \mathbb{R}$, there exist constants $\omega>0, M_{j}^{-}$and $M_{j}^{+}$ such that

$$
\left|f_{j}(x)\right| \leq \omega_{j} \quad \text { and } \quad M_{j}^{-} \leq \frac{\left|f_{j}(x)-f_{j}(y)\right|}{x-y} \leq M_{j}^{+},
$$


(H2) for all $j=1, \ldots, n$, the functions $k_{j}: \mathbb{R}_{+} \rightarrow \mathbb{R}_{+}$are continuous and satisfy

$$
\int_{0}^{+\infty} k_{j}(u) \mathrm{d} u=\mathbf{k}_{j} \text { and } \int_{0}^{+\infty} u k_{j}(u) \mathrm{d} u<\infty
$$

(H3) $C-A^{+} M-B^{+} K^{+} M$ is a M-matrix.

Remark 2.1. Under assumptions ( $\mathrm{H} 1)$ and $(\mathrm{H} 2)$, the existence of the solutions of System [1] is guaranteed by using [53, Theorem 2.1]. It should also be pointed out that the constants $M_{j}^{+}$ and $M_{j}^{-}$can be negative or positive in the assumption (H1) which allows Lurie-type functions if we take $M_{j}^{+}, M_{j}^{-}>0$ [55] or Lipschitz functions if we take $M_{j}^{-}=-M_{j}^{+}<0$. Therefore $(\mathrm{H} 1)$ is weaker than the assumptions used for instance in [56, 57, 58]. Finally, if we only consider Lipschitz activation functions as in [12], the method used in [12] for proving the existence of an equilibrium point does not work for System (1) with the high-order terms.

Definition 2.1. ([[12] $)$ A nonsingular matrix $X=\left(x_{i j}\right)_{n \times n}$ is a $M$-matrix if $X=r I-\beta$ with $\beta \geq 0$ and $r>\rho(\beta)$ where $\rho(\beta)$ stands for the spectral radius of the matrix $\beta$.

Let

$$
Z^{n \times n}=\left\{A=\left(a_{i j}\right) \in \mathbb{R}^{n \times n}: a_{i i}>0, a_{i j} \leq 0, \text { for all } i \neq j\right\}
$$

then we have the following lemma:

Lemma 2.2. ([โ12]) The following claims are equivalent:

(i) there exist $\delta_{j}>0$ such that $\sum_{j=1}^{n} a_{j i} \delta_{j}>0$ for all $i=1, \ldots, n$;

(ii) $A \in Z^{n \times n}$ is a M-matrix;

(iii) there exist $\delta_{j}>0$ such that $\sum_{j=1}^{n} a_{i j} \delta_{j}>0$ for all $i=1, \ldots, n$.

Let us give the definition of the topological degree.

Definition 2.3. ([12] $]$ ) Assume that $F: \Omega \rightarrow \mathbb{R}^{n}$ is a continuously differentiable function where $\Omega \subset \mathbb{R}^{n}$ is an open bounded set. If $J_{F}(u)=0$ for any $u \in F^{-1}(p)$ and $p \notin F(\partial \Omega)$, where $J_{F}$ denotes the Jacobian determinant relative to $F$, then the topological degree relative to $\Omega$ and $p$ is given by

$$
\operatorname{deg}(F, \Omega, p)=\left\{\begin{array}{lll}
\sum_{u \in F^{-1}(p)} \operatorname{sign} J_{F}(u) & \text { if } & F^{-1}(p) \neq \emptyset \\
0 & \text { if } & F^{-1}(p)=\emptyset
\end{array}\right.
$$

Let us introduce the notion of finite time boundedness.

Definition 2.4. ([59]) System (1) is said to be finite time bounded $(F T B)$ with respect to $\left(c_{1}, c_{2}, R, T\right)$ where $0<c_{1} \leq c_{2}, R>0$ and $T>0$ if for all $t \in[0, T]$ we have

$$
\max \left\{\sup _{\theta \leq 0}\left\{x^{T}(\theta) R x(\theta)\right\}, \sup _{-\bar{h} \leq \boldsymbol{\theta} \leq 0}\left\{\dot{x}^{T}(\theta) R \dot{x}(\theta)\right\}\right\} \leq c_{1}
$$

implies that

$$
x^{T}(t) R x(t)<c_{2}
$$

Let us give two lemmas useful to prove our first result.

Lemma 2.5. ([15]) Given any real matrix $M=M^{T}>0$ of appropriate dimension and a vector field $\omega:[a, b] \rightarrow \mathbb{R}^{n}$ such that the integrations concerned are well defined, then we have

$$
\left[\int_{a}^{b} \omega(s) d s\right]^{T} M \int_{a}^{b} \omega(s) d s \leq(b-a) \int_{a}^{b} \omega^{T}(s) M \omega(s) d s .
$$

Lemma 2.6. ([60]) Let $P \in \mathbb{R}^{n \times n}$ be a symmetric matrix, then we have

$$
\lambda_{\min }(P) x^{T} x \leq x^{T} P x \leq \lambda_{\max }(P) x^{T} x
$$

for any $x \in \mathbb{R}^{n}$.

\section{Existence of equilibrium points}

In [15], the existence of equilibrium points for neutral lower order Hopfield NNs with time delay in the leakage term is discussed. Thanks to the high-order terms, the NNs given by (1) are more general. Compared with results in [15], we extend the existence of equilibrium points to the more general class of NHOHNNs.

We introduce the following notations:

$$
\begin{aligned}
M_{j} & =\max \left\{\left|M_{j}^{-}\right|,\left|M_{j}^{+}\right|\right\}, \quad M=\operatorname{diag}\left(M_{1}, \ldots, M_{n}\right) \\
A & =\left[a_{i j}\right]_{n \times n}, \quad B=\left[b_{i j}\right]_{n \times n}, \quad D=\left[d_{i j}\right]_{n \times n} .
\end{aligned}
$$

We present a sufficient condition which guarantee the existence of equilibrium points for the NHOHNNs given by (1).

Theorem 3.1. Under assumptions $(H 1)-(H 2)-(H 3)$, System (1) has at least one equilibrium point.

Proof. If $x^{*}=\left(x_{1}^{*}, \ldots, x_{n}^{*}\right)^{T}$ denotes an equilibrium point of System (1), then $x^{*}$ satisfies for all $i=1, \ldots, n$

$$
\begin{aligned}
& -c_{i} x_{i}^{*}+\sum_{j=1}^{n} a_{i j} f_{j}\left(x_{j}^{*}\right)+\sum_{j=1}^{n} \sum_{k=1}^{n} T_{i j k} f_{k}\left(x_{k}^{*}\right) f_{j}\left(x_{j}^{*}\right) \\
& +\sum_{j=1}^{n} b_{i j} \int_{-\infty}^{t} k_{j}(t-s) f_{j}\left(x_{j}^{*}\right) \mathrm{d} s+\sum_{j=1}^{n} d_{i j} \dot{x}_{j}^{*}+J_{i}=0
\end{aligned}
$$

By using (H2), Equation (2) is equivalent to

$$
\begin{aligned}
& -c_{i} x_{i}^{*}+\sum_{j=1}^{n} a_{i j} f_{j}\left(x_{j}^{*}\right)+\sum_{j=1}^{n} \sum_{k=1}^{n} T_{i j k} f_{k}\left(x_{k}^{*}\right) f_{j}\left(x_{j}^{*}\right) \\
& +\sum_{j=1}^{n} b_{i j} \mathbf{k}_{j} f_{j}\left(x_{j}^{*}\right)+J_{i}=0 .
\end{aligned}
$$

Let

$$
l_{i}(x)=c_{i} x_{i}-\sum_{j=1}^{n}\left(a_{i j}+b_{i j} \mathbf{k}_{j}\right) f_{j}\left(x_{j}\right)-\sum_{j=1}^{n} \sum_{k=1}^{n} T_{i j k} f_{k}\left(x_{k}\right) f_{j}\left(x_{j}\right)-J_{i} .
$$

Clearly, a solution of $l(x)=\left(l_{1}(x), \ldots, l_{n}(x)\right)^{T}=0$ is an equilibrium of System (1). Now, we define the homotopy mapping

$$
F(x, \lambda)=\left(F_{1}(x), \ldots, F_{n}(x)\right)^{T}
$$


where $\lambda \in[0,1]$ and $F_{i}(x)=\lambda l_{i}(x)+(1-\lambda) x_{i}$. It follows from assumptions $(H 1)-(H 2)$ that for all $1 \leq i \leq n$

$$
\begin{aligned}
\left|F_{i}(x, \lambda)\right| & =\mid \lambda\left[c_{i} x_{i}-\sum_{j=1}^{n} a_{i j} f_{j}\left(x_{j}\right)-\sum_{j=1}^{n} \sum_{k=1}^{n} T_{i j k} f_{k}\left(x_{k}\right) f_{j}\left(x_{j}\right)\right. \\
& \left.-\sum_{j=1}^{n} b_{i j} \mathbf{k}_{j} f_{j}\left(x_{j}\right)-J_{i}\right]+(1-\lambda) x_{i} \mid \\
& \geq\left|\lambda c_{i} x_{i}+(1-\lambda) x_{i}\right|-\lambda \sum_{j=1}^{n}\left|a_{i j}\right|\left|f_{j}\left(x_{j}\right)\right| \\
& -\lambda \sum_{j=1}^{n} \sum_{k=1}^{n}\left|T_{i j k}\right|\left|f_{k}\left(x_{k}\right)\right|\left|f_{j}\left(x_{j}\right)\right| \\
& -\lambda \sum_{j=1}^{n}\left|b_{i j}\right|\left|\mathbf{k}_{j}\right|\left|f_{j}\left(x_{j}\right)\right|-\lambda\left|J_{i}\right| \\
& \geq\left|\lambda c_{i} x_{i}+(1-\lambda) x_{i}\right|-\lambda \sum_{j=1}^{n}\left|a_{i j}\right| M_{j}\left|x_{j}\right| \\
& -\lambda \sum_{j=1}^{n}\left|b_{i j}\right|\left|\mathbf{k}_{j}\right| M_{j}\left|x_{j}\right|-\lambda\left[\left|J_{i}\right|+\sum_{j=1}^{n} \sum_{k=1}^{n}\left|T_{i j k}\right| \omega^{2}\right] .
\end{aligned}
$$

Since $C-A^{+} M-B^{+} K^{+} M$ is a M-matrix, Lemma 2.2 implies that there exists constants $\delta_{i}>0$ with $i=1, \ldots, n$ such that

$$
\delta_{i} c_{i}-\sum_{j=1}^{n} \delta_{j}\left|a_{i j}\right| M_{j}-\sum_{j=1}^{n} \delta_{j}\left|b_{i j}\right|\left|\mathbf{k}_{j}\right| M_{j}>0
$$

or

$$
\delta_{i} c_{i}-\sum_{j=1}^{n} \delta_{j}\left|a_{j i}\right| M_{i}-\sum_{j=1}^{n} \delta_{j}\left|b_{j i}\right|\left|\mathbf{k}_{i}\right| M_{i}>0 .
$$

For $F(x)=\left(F_{1}\left(x_{1}\right), \ldots, F_{n}\left(x_{n}\right)\right)^{T}$, we have

$$
\begin{aligned}
\sum_{i=1}^{n} \delta_{i}\left|F_{i}(x, \lambda)\right| & \geq \sum_{i=1}^{n} \delta_{i}(1-\lambda)\left|x_{i}\right|+\lambda \sum_{i=1}^{n}\left[\delta_{i} c_{i}\left|x_{i}\right|\right. \\
& \left.-\delta_{i} \sum_{j=1}^{n}\left|a_{i j}\right| M_{j}\left|x_{j}\right|-\delta_{i} \sum_{j=1}^{n}\left|b_{i j}\right|\left|\mathbf{k}_{j}\right| M_{j}\left|x_{j}\right|\right] \\
& -\lambda \sum_{i=1}^{n} \delta_{i}\left(\left|J_{i}\right|+\sum_{j=1}^{n} \sum_{k=1}^{n}\left|T_{i j k}\right| \omega^{2}\right) \\
& \geq \lambda \sum_{i=1}^{n}\left[\delta_{i} c_{i}\left|x_{i}\right|-\delta_{i} \sum_{j=1}^{n} M_{j}\left|a_{i j}\right|\left|x_{j}\right|\right. \\
& \left.-\delta_{i} \sum_{j=1}^{n} M_{j}\left|b_{i j}\right|\left|\mathbf{k}_{j}\right|\left|x_{j}\right|\right] \\
& -\lambda \sum_{i=1}^{n} \delta_{i}\left(\left|J_{i}\right|+\sum_{j=1}^{n} \sum_{k=1}^{n}\left|T_{i j k}\right| \omega^{2}\right) \\
& =\lambda \sum_{i=1}^{n}\left[\delta_{i} c_{i}-\sum_{j=1}^{n} \delta_{j}\left|a_{j i}\right| M_{i}\right. \\
& \left.-\sum_{j=1}^{n} \delta_{j}\left|b_{j i}\right| M_{i}\left|\mathbf{k}_{i}\right|\right]\left|x_{i}\right| \\
& -\lambda \sum_{i=1}^{n} \delta_{i}\left(\left|J_{i}\right|+\sum_{j=1}^{n} \sum_{k=1}^{n}\left|T_{i j k}\right| \omega^{2}\right)
\end{aligned}
$$

Define

$$
\begin{aligned}
& \delta_{0}=\min _{1 \leq i \leq n}\left\{\delta_{i} c_{i}-\sum_{j=1}^{n} \delta_{j}\left|a_{j i}\right| M_{i}-\sum_{j=1}^{n} \delta_{j}\left|b_{j i}\right| M_{i}\left|\mathbf{k}_{i}\right|\right\} \\
& \Delta_{0}=\max _{1 \leq i \leq n}\left\{\delta_{i}\left(\left|J_{i}\right|+\sum_{j=1}^{n} \sum_{k=1}^{n}\left|T_{i j k}\right| \omega^{2}\right)\right\}
\end{aligned}
$$

Let

$$
\Omega=\left\{x:\left|x_{i}\right|<\beta=\frac{n\left(\Delta_{0}+1\right)}{\delta_{0}}\right\}
$$

then $\Omega$ is non-empty from (4). It follows from (5) that for any $x \in \partial \Omega$ there exists $1 \leq i_{0} \leq n$ such that $\left|x_{i_{0}}\right|=\beta$. So we have

$$
\begin{aligned}
\sum_{i=1}^{n} \delta_{i}\left|F_{i}(x, \lambda)\right| & \geq \lambda \sum_{i=1}^{n}\left[\delta_{i_{0}} c_{i_{0}}-\sum_{j=1}^{n} \delta_{j} M_{i_{0}}\left|a_{j i_{0}}\right|\right. \\
& \left.-\sum_{j=1}^{n} \delta_{j} M_{i_{0}}\left|b_{j i_{0}}\right|\left|\mathbf{k}_{i_{0}}\right|\right]\left|x_{i_{0}}\right|-\lambda \sum_{i=1}^{n} \Delta_{0} \\
& \geq \lambda \delta_{0}\left|x_{i_{0}}\right|-\lambda n \Delta_{0}>0 .
\end{aligned}
$$

For all $\lambda \in(0,1]$, it means that $F(x, \lambda) \neq 0$ for any $x \in \partial \Omega$ and $\lambda \in(0,1]$. If $\lambda=0$, we have $F(x, \lambda)=x \neq 0$ for any $x \in \partial \Omega$. Therefore $F(x, \lambda) \neq 0$, for any $x \in \partial \Omega, \lambda \in[0,1]$. We have $\operatorname{deg}\left(I_{d}, \Omega, 0\right)=1$ where $I_{d}(x)=x$ and $\operatorname{deg}\left(I_{d}, \Omega, 0\right)$ is the topological degree. Thus from the homotopy invariance theorem given for instance in [61, page 13], we obtain

$$
\operatorname{deg}\left(I_{d}, \Omega, 0\right)=\operatorname{deg}(l(.), \Omega, 0)=1 .
$$

By using the topological degree theory [61], we can conclude that the equation $l(x)=0$ has at least one solution in $\Omega$. It implies that System (1) has at least one equilibrium point.

\section{Finite Time boundedness analysis}

\subsection{Finite time Boundedness}

Compared with some existing results in [15, 24, 42], System (1) is more general to some extent. Moreover, the usual Lyapunov stability is studied in [24, 15] whereas the concept of FTB is discussed in this section. Assume that $x^{*}=\left(x_{1}^{*}, \ldots, x_{n}^{*}\right)^{T}$ is an equilibrium point of System (1). By a simple transformation

$$
z(t)=x(t)-x^{*}
$$

we can shift the equilibrium point $x^{*}$ to the origin. By using (2), System (1) can be rewritten as (see [62]):

$$
\left\{\begin{aligned}
\dot{z}(t) & =-C z(t-\sigma)+\left(A+\Gamma^{T} T^{*}\right) g(z(t-\tau(t))) \\
& +B \int_{-\infty}^{t} K(t-s) g(z(s)) \mathrm{d} s+D \dot{z}(t-h(t)) \\
z(s) & =\phi(s)-x^{*}, \quad s \in(-\infty, 0]
\end{aligned}\right.
$$

where

$$
\begin{aligned}
g(z(.)) & =f\left(z(.)+x^{*}\right)-f\left(x^{*}\right), \quad T_{i}=\left[T_{i j k}\right]_{n \times n}, \\
T^{*} & =\left[T_{1}+T_{1}^{T}, \ldots, T_{n}+T_{n}^{T}\right]^{T}, \\
\Gamma & =\operatorname{diag}[\xi, \ldots, \xi], \xi=\left[\xi_{1}, \ldots, \xi_{n}\right]^{T}, \\
\xi_{i} & =\frac{T_{i j k}}{T_{i j k}+T_{i k j}} f_{k}\left(x_{k}(t-\tau(t))+\frac{T_{i k j}}{T_{i j k}+T_{i k j}} f_{k}\left(x_{k}^{*}\right) .\right.
\end{aligned}
$$


We will use this $z$-form of System (1) for the proof of the results of our article. We introduce the following notations:

$$
\Gamma^{+}=\operatorname{diag}\left[\xi^{+}, \ldots, \xi^{+}\right], \xi^{+}=\left[\omega_{1}, \ldots, \omega_{n}\right]^{T}, c^{+}=\max _{1 \leq i \leq n} c_{i} .
$$

The methods used in [47, 48, 42, 63, 64] for ensuring the FTB of NNs require the differentiability and the boundedness of the derivative of the time-varying delays. For improving these results, we remove this restriction by establishing the following theorem where the time-varying delays are not necessary differentiable.

Theorem 4.1. Under assumptions $(H 1)-(H 2)-(H 3)$, System (1) is FTB with respect to $\left(c_{1}, c_{2}, R, T\right)$ if there exist a positive scalar $\alpha$, two $n \times n$ matrices $Q_{1}, Q_{2}$, three $n \times n$ symmetric positive definite matrices $P, Q_{3}, Q_{4}$, four $n \times n$ positive diagonal matrices $U_{1}, U_{2}, Q_{5}, Q_{6}$, and a $2 n \times 2 n$ matrix

$$
Q_{7}=\left[\begin{array}{cc}
T_{11} & T_{12} \\
* & T_{22}
\end{array}\right]>0
$$

such that the following conditions hold:

$$
\Xi=\left[\begin{array}{ccccccccc}
\Pi_{11} & 0 & \Pi_{13} & \Pi_{14} & 0 & \Pi_{16} & \Pi_{17} & \Pi_{18} & \Pi_{19} \\
* & \Pi_{22} & \Pi_{23} & 0 & \Pi_{25} & 0 & 0 & \Pi_{28} & \Pi_{29} \\
* & * & \Pi_{33} & 0 & \Pi_{35} & \Pi_{36} & 0 & \Pi_{38} & \Pi_{39} \\
* & * & * & \Pi_{44} & 0 & 0 & 0 & \Pi_{48} & 0 \\
* & * & * & * & \Pi_{55} & 0 & 0 & 0 & 0 \\
* & * & * & * & * & \Pi_{66} & 0 & \Pi_{68} & \Pi_{69} \\
* & * & * & * & * & * & \Pi_{77} & 0 & 0 \\
* & * & * & * & * & * & * & \Pi_{88} & 0 \\
* & * & * & * & * & * & * & * & \Pi_{99}
\end{array}\right]<0 \quad(6)
$$

and

$$
\left[\begin{array}{cc}
c_{2} e^{-\alpha T} & \sqrt{c_{1}} \\
\sqrt{c_{1}} & \omega_{1}^{-1}
\end{array}\right]>0
$$

where

$$
\begin{aligned}
& \Pi_{11}=-P C-C P+Q_{3}+\sigma^{2} Q_{4}-U_{1} \Sigma_{1}-\alpha P, \\
& \Pi_{13}=P D, \quad \Pi_{14}=T_{12}^{T}, \quad \Pi_{16}=C P C+\alpha C P, \\
& \Pi_{17}=U_{1} \Sigma_{2}, \quad \Pi_{18}=P \tilde{A}, \quad \Pi_{19}=P B, \\
& \Pi_{22}=\tau T_{22}+Q_{6}-Q_{1}-Q_{1}^{T}, \quad \Pi_{23}=Q_{1} D+Q_{2}^{T} D, \\
& \Pi_{25}=-Q_{1} C, \quad \Pi_{28}=Q_{1} \tilde{A}, \quad \Pi_{29}=Q_{1} B, \\
& \Pi_{33}=-Q_{6}\left(1-h^{*}\right)-D^{T} Q_{2} D-D^{T} Q_{2}^{T} D, \quad \Pi_{35}=D^{T} Q_{2} C, \\
& \Pi_{36}=-D^{T} P C, \quad \Pi_{38}=-D^{T} Q_{2} \tilde{A}, \quad \Pi_{39}=-D^{T} Q_{2} B, \\
& \Pi_{44}=\tau T_{11}-T_{12}-T_{12}^{T}-U_{2} \Sigma_{1}, \quad \Pi_{48}=U_{2} \Sigma_{2}, \quad \Pi_{55}=-Q_{3}, \\
& \Pi_{66}=-Q_{4}-\alpha C P C, \quad \Pi_{68}=-C P \tilde{A}, \quad \Pi_{69}=-C P B, \\
& \Pi_{77}=Q_{5} \kappa-U_{1}, \quad \Pi_{88}=-U_{2}, \quad \Pi_{99}=-Q_{5},
\end{aligned}
$$

and

$$
\begin{aligned}
\omega_{1} & =\left[2 \lambda_{\max }(P)\left(1+\sigma^{2} c^{+^{2}}\right)+\sigma \lambda_{\max }\left(Q_{3}\right)\right. \\
& +\bar{h} \lambda_{\max }\left(Q_{6}\right)+\sigma^{3} \lambda_{\max }\left(Q_{4}\right)+\tau^{2} \lambda_{\max }\left(T_{22}\right) \\
& \left.+\sum_{j=1}^{n} q_{j} \mathbf{k}_{j} \max _{j} M_{j}^{2} \int_{0}^{\infty} u k_{j}(u) \mathrm{d} u\right] \\
& \times\left[\left(\frac{n c^{+} \sigma}{\lambda_{\min }\left(Q_{3}\right)}\right)^{\frac{1}{2}}+\left(\lambda_{\min }(P)\right)^{\frac{-1}{2}}\right]^{2} \operatorname{cond}(P) \operatorname{cond}(\tilde{P})
\end{aligned}
$$

with

$$
\begin{aligned}
\Sigma_{1} & =\operatorname{diag}\left(M_{1}^{-} M_{1}^{+}, \ldots, M_{n}^{-} M_{n}^{+}\right), \\
\Sigma_{2} & =\operatorname{diag}\left(\frac{M_{1}^{-}+M_{1}^{+}}{2}, \ldots, \frac{M_{n}^{-}+M_{n}^{+}}{2}\right), \\
Q_{5} & =\operatorname{diag}\left(q_{1}, \ldots, q_{n}\right), \quad \kappa=\operatorname{diag}\left(\mathbf{k}_{1}^{2}, \ldots, \mathbf{k}_{n}^{2}\right), \\
\tilde{A} & =A+\Gamma^{+^{T}} T^{*}, \quad \tilde{P}=R^{\frac{1}{2}} P R^{\frac{1}{2}}, \\
\operatorname{cond}(P) & =\frac{\lambda_{\max }(P)}{\lambda_{\min }(P)} \text { the condition number of } P .
\end{aligned}
$$

The proof of Theorem 4.1 is inspired by the proof of Theorem 2 in [15].

PROOF. Let us consider the following LKF

$$
V(t, z(t))=\sum_{i=1}^{6} V_{i}(t, z(t))
$$

where

$$
\begin{aligned}
& V_{1}(t, z(t))=\left[z(t)-C \int_{t-\sigma}^{t} z(s) \mathrm{d} s\right]^{T} P\left[z(t)-C \int_{t-\sigma}^{t} z(s) \mathrm{d} s\right] \\
& V_{2}(t, z(t))=\int_{t-\sigma}^{t} z^{T}(s) Q_{3} z(s) \mathrm{d} s+\int_{t-h(t)}^{t} \dot{z}^{T}(s) Q_{6} \dot{z}(s) \mathrm{d} s \\
& V_{3}(t, z(t))=\sigma \int_{t-\sigma}^{t} \int_{s}^{t} z^{T}(u) Q_{4} z(u) \mathrm{d} u \mathrm{~d} s \\
& V_{4}(t, z(t))=\int_{0}^{t} \int_{u-\tau(u)}^{u}\left[\begin{array}{c}
z(u-\tau(u)) \\
\dot{z}(s)
\end{array}\right]^{T} Q_{7}\left[\begin{array}{c}
z(u-\tau(u)) \\
\dot{z}(s)
\end{array}\right] \mathrm{d} s \mathrm{~d} u \\
& V_{5}(t, z(t))=\int_{-\tau}^{0} \int_{t+u}^{t} \dot{z}^{T}(s) T_{22} \dot{z}(s) \mathrm{d} s \mathrm{~d} u \\
& V_{6}(t, z(t))=\sum_{j=1}^{n} q_{j} \mathbf{k}_{j} \int_{0}^{\infty} k_{j}(u) \int_{t-u}^{t} g_{j}^{2}\left(z_{j}(s)\right) \mathrm{d} s \mathrm{~d} u
\end{aligned}
$$

By calculating $\dot{V}(t, z(t))$ (see AppendixA, we obtain

$$
\dot{V}(t, z(t)) \leq \zeta^{T}(t, z(t)) \bar{\Xi} \zeta(t, z(t))
$$

where

$$
\begin{aligned}
\zeta(t, z(t))= & {\left[z^{T}(t), \dot{z}^{T}(t), \dot{z}^{T}(t-h(t)), z^{T}(t-\tau(t)), z^{T}(t-\sigma),\right.} \\
& \left(\int_{t-\sigma}^{t} z(s) d s\right)^{T}, g^{T}(z(t)), g^{T}(z(t-\tau(t))), \\
& \left.\left(\int_{-\infty}^{t} K(t-s) g(z(s)) d s\right)^{T}\right]^{T}
\end{aligned}
$$

and

$$
\bar{\Xi}=\left[\begin{array}{ccccccccc}
\bar{\Pi}_{11} & 0 & \Pi_{13} & \Pi_{14} & 0 & \bar{\Pi}_{16} & \Pi_{17} & \Pi_{18} & \Pi_{19} \\
* & \Pi_{22} & \Pi_{23} & 0 & \Pi_{25} & 0 & 0 & \Pi_{28} & \Pi_{29} \\
* & * & \Pi_{33} & 0 & \Pi_{35} & \Pi_{36} & 0 & \Pi_{38} & \Pi_{39} \\
* & * & * & \Pi_{44} & 0 & 0 & 0 & \Pi_{48} & 0 \\
* & * & * & * & \Pi_{55} & 0 & 0 & 0 & 0 \\
* & * & * & * & * & \bar{\Pi}_{66} & 0 & \Pi_{68} & \Pi_{69} \\
* & * & * & * & * & * & \Pi_{77} & 0 & 0 \\
* & * & * & * & * & * & * & \Pi_{88} & 0 \\
* & * & * & * & * & * & * & * & \Pi_{99}
\end{array}\right]<0 \quad(9)
$$


with

$\bar{\Pi}_{11}=-P C-C P+Q_{3}+\sigma^{2} Q_{4}-U_{1} \Sigma_{1}, \bar{\Pi}_{16}=C P C, \bar{\Pi}_{66}=-Q_{4}$.

Therefore

$$
\begin{aligned}
\dot{V}(t, z(t)) & \leq \zeta^{T}(t, z(t)) \bar{\Xi} \zeta(t, z(t)) \\
& \leq \zeta^{T}(t, z(t)) \Xi \zeta(t, z(t))+\alpha V_{1}(t, z(t)) \\
& \leq \zeta^{T}(t, z(t)) \Xi \zeta(t, z(t))+\alpha V(t, z(t))
\end{aligned}
$$

with

$$
\bar{\Xi}=\Xi+\left[\begin{array}{ccccccccc}
\alpha P & 0 & 0 & 0 & 0 & -\alpha C P & 0 & 0 & 0 \\
* & 0 & 0 & 0 & 0 & 0 & 0 & 0 & 0 \\
* & * & 0 & 0 & 0 & 0 & 0 & 0 & 0 \\
* & * & * & 0 & 0 & 0 & 0 & 0 & 0 \\
* & * & * & * & 0 & 0 & 0 & 0 & 0 \\
* & * & * & * & * & \alpha C P C & 0 & 0 & 0 \\
* & * & * & * & * & * & 0 & 0 & 0 \\
* & * & * & * & * & * & * & 0 & 0 \\
* & * & * & * & * & * & * & * & 0
\end{array}\right]
$$

Since $\Xi<0$, it leads to

$$
\dot{V}(t, z(t)) \leq \alpha V(t, z(t)) .
$$

Integrating 10$]$ from 0 to $t \in[0, T]$, we obtain

$$
V(t, z(t)) \leq e^{\alpha t} V(z(0))
$$

where

$$
\begin{aligned}
V(z(0)) \leq & {\left[2 \lambda_{\max }(P)\left(1+\sigma^{2} c^{+2}\right)+\sigma \lambda_{\max }\left(Q_{3}\right)+\bar{h} \lambda_{\max }\left(Q_{6}\right)\right.} \\
& +\sigma^{3} \lambda_{\max }\left(Q_{4}\right)+\tau^{2} \lambda_{\max }\left(T_{22}\right) \\
& \left.+\sum_{j=1}^{n} q_{j} \mathbf{k}_{j} \max _{j} M_{j}^{2} \int_{0}^{\infty} u k_{j}(u) \mathrm{d} u\right]\|\phi\|_{h}^{2}
\end{aligned}
$$

On one hand, by using the Cauchy-Schwartz inequality and Lemma 2.6 we have

$$
\begin{aligned}
\|z(t)\| & \leq\left\|C \int_{t-\sigma}^{t} z(s) \mathrm{d} s\right\|+\sqrt{\frac{V_{1}(t, z(t))}{\lambda_{\min }(P)}} \\
& \leq\left\|C \int_{t-\sigma}^{t} z(s) \mathrm{d} s\right\|+\sqrt{\frac{V(t, z(t))}{\lambda_{\min }(P)}}
\end{aligned}
$$

and since

$$
\begin{aligned}
\left\|\int_{t-\sigma}^{t} z(s) \mathrm{d} s\right\|^{2} & =\left(\int_{t-\sigma}^{t} z(s) \mathrm{d} s\right)^{T}\left(\int_{t-\sigma}^{t} z(s) \mathrm{d} s\right) \\
& \leq \sigma \int_{t-\sigma}^{t} z^{T}(s) z(s) \mathrm{d} s \\
& \leq \frac{\sigma}{\lambda_{\min }\left(Q_{3}\right)} \int_{t-\sigma}^{t} z^{T}(s) Q_{3} z(s) \mathrm{d} s \\
& \leq \frac{\sigma}{\lambda_{\min }\left(Q_{3}\right)} V(t, z(t))
\end{aligned}
$$

we obtain

$$
\|z(t)\| \leq\left[\left(\frac{n c^{+} \sigma}{\lambda_{\min }\left(Q_{3}\right)}\right)^{\frac{1}{2}}+\left(\lambda_{\min }(P)\right)^{\frac{-1}{2}}\right] \sqrt{V(t)} .
$$

On the other hand, Lemma 2.6, implies that

$$
\begin{aligned}
& \lambda_{\max }(\tilde{P})\|z(t)\|^{2} \geq z^{T}(t) \tilde{P} z(t) \geq \lambda_{\min }(P) z^{T}(t) R z(t), \\
& \lambda_{\min }(\tilde{P})\|z(0)\|^{2} \leq z^{T}(0) \tilde{P} z(0) \leq \lambda_{\max }(P) z^{T}(0) R z(0) .
\end{aligned}
$$

So (11)-(12)-(14) and 15 prove that

$$
\begin{aligned}
\|z(t)\| & \leq\left[\left(\frac{n c^{+} \sigma}{\lambda_{\min }\left(Q_{3}\right)}\right)^{\frac{1}{2}}+\left(\lambda_{\min }(P)\right)^{\frac{-1}{2}}\right] e^{\frac{\alpha}{2} T} \sqrt{V(z((0))} \\
& \leq e^{\frac{\alpha}{2} T}\left[2 \lambda_{\max }(P)\left(1+\sigma^{2} c^{+^{2}}\right)+\sigma \lambda_{\max }\left(Q_{3}\right)\right. \\
& +\bar{h} \lambda_{\max }\left(Q_{6}\right)+\sigma^{3} \lambda_{\max }\left(Q_{4}\right)+\tau^{2} \lambda_{\max }\left(T_{22}\right) \\
& \left.+\sum_{j=1}^{n} q_{j} \mathbf{k}_{j} \max _{j} M_{j}^{2} \int_{0}^{\infty} u k_{j}(u) \mathrm{d} u\right]^{\frac{1}{2}} \\
& \times\left[\left(\frac{n c^{+} \sigma}{\lambda_{\min }\left(Q_{3}\right)}\right)^{\frac{1}{2}}+\left(\lambda_{\min }(P)\right)^{\frac{-1}{2}}\right] \sqrt{\frac{\lambda_{\max }(P)}{\lambda_{\min }(\tilde{P})} c_{1}}
\end{aligned}
$$

and

$$
\begin{aligned}
z^{T}(t) R z(t) & \leq e^{\alpha T}\left[2 \lambda_{\max }(P)\left(1+\sigma^{2} c^{+^{2}}\right)+\sigma \lambda_{\max }\left(Q_{3}\right)\right. \\
& +\bar{h} \lambda_{\max }\left(Q_{6}\right)+\sigma^{3} \lambda_{\max }\left(Q_{4}\right)+\tau^{2} \lambda_{\max }\left(T_{22}\right) \\
& \left.+\sum_{j=1}^{n} q_{j} \mathbf{k}_{j} \max _{j} M_{j}^{2} \int_{0}^{\infty} u k_{j}(u) \mathrm{d} u\right] \\
& \times\left[\left(\frac{n c^{+} \sigma}{\lambda_{\min }\left(Q_{3}\right)}\right)^{\frac{1}{2}}+\left(\lambda_{\min }(P)\right)^{\frac{-1}{2}}\right]^{2} \\
& \times \operatorname{cond}(P) \operatorname{cond}(\tilde{P}) c_{1}
\end{aligned}
$$

The inequality (17) and the Schur complement lemma, given for instance in [65], applied to condition (7) prove that

$$
z(t)^{T} R z(t)<c_{2} \text { for all } t \in[0, T] .
$$

So, the proof is completed.

Remark 4.1. If conditions (6) and (7) of Theorem 1 are satisfied with $\alpha=0$, then System (1) is asymptotically stable in the sense of Lyapunov. The LKF inequality $(10)$ is used to deal with the problem of FTB of the NHOHNNs with infinite distributed delays and leakage delay. Compared with [15], some new sufficient conditions in terms of LMIs are established to make our results less conservative with respect to the delays. When the number of neurons $n$ increases, the complexity increases strongly because $8.5 n^{2}+4 n+1$ variables are involved in the LMIs that must be resolved. Such a complexity is caused by the use of the LKF inequality 10 .

Remark 4.2. Condition (7) is not standard LMIs, however it 
can be guaranteed by the following conditions

$$
\begin{gathered}
\lambda_{1} I<P<\lambda_{2} I, \lambda_{3} I<Q_{3}<\lambda_{4} I, Q_{4}<\lambda_{5} I, Q_{6}<\lambda_{6} I, T_{22}<\lambda_{7} I \\
-c_{2} r_{1}^{2} \lambda_{1}^{2} e^{-\alpha T}+c_{1}\left[2 \lambda_{2}\left(1+\sigma^{2} c^{+^{2}}\right)+\sigma \lambda_{4}+\bar{h} \lambda_{6}+\sigma^{3} \lambda_{5}+\tau^{2} \lambda_{7}\right. \\
\left.\quad+\sum_{j=1}^{n} q_{j} \mathbf{k}_{j} \max _{j} M_{j}^{2} \int_{0}^{\infty} u k_{j}(u) d u\right]\left[\frac{n c^{+} \sigma}{\sqrt{\lambda_{3}}}+\frac{1}{\sqrt{\lambda_{1}}}\right]^{2} r_{2}^{2} \lambda_{2}^{2}<0
\end{gathered}
$$

where $r_{1}=\lambda_{\min }(R), r_{2}=\lambda_{\max }(R)$ and $\lambda_{i}, i=1, \ldots 7$ are unknown positive variables. It should be pointed out that $(19)$ is not a LMI w.r.t. $\lambda_{i}, i=1,2,3$ because $\lambda_{i}$ appears in a nonlinear fashion. Therefore, we first find the scalars $\lambda_{i}$ from (6) and then we solve (19) which then becomes a LMI.

When $\tau(t)=0$ and $K=0$, System (1) turns into

$$
\left\{\begin{aligned}
\dot{x}_{i}(t) & =-c_{i} x_{i}(t-\sigma)+\sum_{j=1}^{n} a_{i j} f_{j}\left(x_{j}(t)\right) \\
& +\sum_{j=1}^{n} \sum_{k=1}^{n} T_{i j k} f_{k}\left(x_{k}(t)\right) f_{j}\left(x_{j}(t)\right) \\
& +\sum_{j=1}^{n} d_{i j} \dot{x}_{j}(t-h(t)) \\
x(s) \quad & \phi(s), \quad s \in(-\infty, 0]
\end{aligned}\right.
$$

Let us give the definition of finite time boundedness for System 20.

Definition 4.2. ([44]) System (20) is said to be Finite Time Bounded (FTB) with respect to $\left(c_{1}, c_{2}, R, T\right), 0<c_{1} \leq c_{2}, T>0$, $R>0$ if for all $t \in[0, T]$

$$
\max \left\{\sup _{-\sigma<\theta \leq 0}\left\{x^{T}(\theta) R x(\theta)\right\}, \sup _{-\bar{h} \leq \theta \leq 0}\left\{\dot{x}^{T}(\theta) R \dot{x}(\theta)\right\}\right\} \leq c_{1}
$$

implies that

$$
x^{T}(t) R x(t)<c_{2}
$$
lary.

According to Definition 4.2 , we deduce the following corol-

Corollary 4.3. Under the assumptions and notations of Theorem 4.1. System (20) is FTB w.r.t. $\left(c_{1}, c_{2}, R, T\right)$ if the following conditions hold:

$$
\tilde{\Xi}=\left[\begin{array}{cccccc}
\tilde{\Pi}_{11} & 0 & \Pi_{13} & 0 & \Pi_{16} & \tilde{\Pi}_{17} \\
* & \tilde{\Pi}_{22} & \Pi_{23} & \Pi_{25} & 0 & 0 \\
* & * & \Pi_{33} & \Pi_{35} & \Pi_{36} & 0 \\
* & * & * & \Pi_{55} & 0 & 0 \\
* & * & * & * & \Pi_{66} & 0 \\
* & * & * & * & * & -U_{1}
\end{array}\right]<0
$$

and

$$
\left[\begin{array}{cc}
c_{2} e^{-\alpha T} & \sqrt{c_{1}} \\
\sqrt{c_{1}} & \omega_{2}^{-1}
\end{array}\right]>0
$$

where

$$
\begin{aligned}
& \tilde{\Pi}_{11}=-P C-C P+Q_{3}+\sigma^{2} Q_{4}-U_{1} \Sigma_{1}-U_{2} \Sigma_{1}-\alpha P, \\
& \tilde{\Pi}_{17}=\left(U_{1}+U_{2}\right) \Sigma_{2}+P \tilde{A}, \quad \tilde{\Pi}_{22}=Q_{6}-Q_{1}-Q_{1}^{T} .
\end{aligned}
$$

and

$$
\begin{aligned}
\omega_{2} & =e^{\alpha T}\left[2 \lambda_{\max }(P)\left(1+\sigma^{2} c^{+^{2}}\right)+\sigma \lambda_{\max }\left(Q_{3}\right)+\bar{h} \lambda_{\max }\left(Q_{6}\right)\right. \\
& \left.+\sigma^{3} \lambda_{\max }\left(Q_{4}\right)\right]\left[\left(\frac{n c^{+} \sigma}{\lambda_{\min }\left(Q_{3}\right)}\right)^{\frac{1}{2}}+\left(\lambda_{\min }(P)\right)^{\frac{-1}{2}}\right]^{2} \\
& \times \operatorname{cond}(P) \operatorname{cond}(\tilde{P}) .
\end{aligned}
$$

Proof. Consider the following LKF

$$
V(t, z(t))=\sum_{i=1}^{3} V_{i}(t, z(t))
$$

By using the LKF 23 and similar arguments to the ones of Theorem 4.1 we obtain easily the result.

Remark 4.3. The lower order class of Hopfield NNs is investigated by many authors [15, 16, 17] and can be considered as a theoretical basis for solving optimization problems. As reported in [66], this class of NNs is expected to produce the poorest quality of solution with a great complexity as measured by the order of the network. Therefore, our work offers a theoretical basis for the design of the second-order class of NNs with mixed time delays more effective in the resolution of optimization problems thanks to the second order synaptic terms $T_{i j k}$.

Now, when the high-order terms $T_{i j k}=0$, System (1) turns into

$$
\left\{\begin{aligned}
\dot{x}_{i}(t) & =-c_{i} x_{i}(t-\sigma)+\sum_{j=1}^{n} a_{i j} f_{j}\left(x_{j}(t-\tau(t))\right) \\
& +\sum_{j=1}^{n} b_{i j} \int_{-\infty}^{t} k_{j}(t-s) f_{j}(x(s)) d s+\sum_{j=1}^{n} d_{i j} \dot{x}_{j}(t-h(t)) \\
x(s) & =\phi(s), \quad s \in(-\infty, 0]
\end{aligned}\right.
$$

Then, we have the following result.

Corollary 4.4. Under the assumptions and notations of Theorem 4.1, System 24) is FTB w.r.t. $\left(c_{1}, c_{2}, R, T\right)$ if the following conditions hold:

$$
\hat{\Xi}=\left[\begin{array}{ccccccccc}
\Pi_{11} & 0 & \Pi_{13} & \Pi_{14} & 0 & \Pi_{16} & \Pi_{17} & \hat{\Pi}_{18} & \Pi_{19} \\
* & \Pi_{22} & \Pi_{23} & 0 & \Pi_{25} & 0 & 0 & \hat{\Pi}_{28} & \Pi_{29} \\
* & * & \Pi_{33} & 0 & \Pi_{35} & \Pi_{36} & 0 & \hat{\Pi}_{38} & \Pi_{39} \\
* & * & * & \Pi_{44} & 0 & 0 & 0 & \Pi_{48} & 0 \\
* & * & * & * & \Pi_{55} & 0 & 0 & 0 & 0 \\
* & * & * & * & * & \Pi_{66} & 0 & \hat{\Pi}_{68} & \Pi_{69} \\
* & * & * & * & * & * & \Pi_{77} & 0 & 0 \\
* & * & * & * & * & * & * & \Pi_{88} & 0 \\
* & * & * & * & * & * & * & * & \Pi_{99}
\end{array}\right]<0
$$

and

$$
\left[\begin{array}{cc}
c_{2} e^{-\alpha T} & \sqrt{c_{1}} \\
\sqrt{c_{1}} & \omega_{1}^{-1}
\end{array}\right]>0
$$

where $\hat{\Pi}_{i 8}=\Pi_{i 8}-P \Gamma^{+^{T}} T^{*}$ for $i=1,2,3,6$. 
The proof of Corollary 4.4 is similar to the one that of Theorem 4.1, so it is omitted.

The computation can be simplified by reducing the parameters involved in the LKF. It is also possible to replace the LKF by a Lyapunov function with the Razumukhin techniques. However, this will have a great influence on the conservatism with respect to the delays, in particular on the upper bounds of the delays [67, 68].

In order to simplify the calculations without losing the conservatism with respect to the delays, we establish the following Corollary 4.5 which provides a simplified criterion for NHOHNNs without delay in the leakage term. This criterion is based on the Schur complement and has been presented for the Lyapunov stability of a special class of NHOHNNs in [69]. The impact of this criterion on the conservatism with respect to the delays will be illustrated in Example 5.3

Corollary 4.5. Under the assumptions and notations of Theorem 4.1. System (1) with $\sigma=0$ is FTB w.r.t. $\left(c_{1}, c_{2}, T, R\right)$ if the following conditions hold:

$\Lambda=\left[\begin{array}{cccccc}-\Pi_{22} & -\Pi_{23} & 0 & 0 & -\Pi_{28} & -\Pi_{29} \\ * & -\Pi_{33} & 0 & 0 & -\Pi_{38} & -\Pi_{39} \\ * & * & -\Pi_{44} & 0 & -\Pi_{48} & 0 \\ * & * & * & -\Pi_{77} & 0 & 0 \\ * & * & * & * & -\Pi_{88} & 0 \\ * & * & * & * & * & -\Pi_{99}\end{array}\right]>0$

and

$$
\left[\begin{array}{cc}
c_{2} e^{-\alpha T} & \sqrt{c_{1}} \\
\sqrt{c_{1}} & \bar{\omega}_{1}
\end{array}\right]>0
$$

where

$$
\begin{aligned}
\bar{P} & =R^{-\frac{1}{2}} P R^{-\frac{1}{2}} \\
\bar{\omega}_{1} & =\frac{\lambda_{\min }(P)}{\operatorname{cond}(\bar{P})}\left[2 \lambda_{\max }(P)+\bar{h} \lambda_{\max }\left(Q_{6}\right)+\tau^{2} \lambda_{\max }\left(T_{22}\right)\right. \\
& \left.+\sum_{j=1}^{n} q_{j} \mathbf{k}_{j} \max _{j} M_{j}^{2} \int_{0}^{\infty} u k_{j}(u) d u\right]^{-1} .
\end{aligned}
$$

PROOF. Let us consider the following LKF

$$
V_{0}(t, z(t))=\sum_{i=1}^{2} \bar{V}_{i}(t, z(t))+\sum_{i=4}^{6} V_{i}(t, z(t))
$$

where

$$
\begin{aligned}
& \bar{V}_{1}(t, z(t))=z(t)^{T} P z(t), \\
& \bar{V}_{2}(t, z(t))=\int_{t-h(t)}^{t} \dot{z}^{T}(s) Q_{6} \dot{z}(s) \mathrm{d} s, \\
& V_{4}(t, z(t))=\int_{0}^{t} \int_{u-\tau(u)}^{u}\left[\begin{array}{c}
z(u-\tau(u)) \\
\dot{z}(s)
\end{array}\right]^{T} Q_{7}\left[\begin{array}{c}
z(u-\tau(u)) \\
\dot{z}(s)
\end{array}\right] \mathrm{d} s \mathrm{~d} u ; \\
& V_{5}(t, z(t))=\int_{-\tau}^{0} \int_{t+u}^{t} \dot{z}^{T}(s) T_{22} \dot{z}(s) \mathrm{d} s \mathrm{~d} u, \\
& V_{6}(t, z(t))=\sum_{j=1}^{n} q_{j} \mathbf{k}_{j} \int_{0}^{\infty} k_{j}(u) \int_{t-u}^{t} g_{j}^{2}\left(z_{j}(s)\right) d s d u .
\end{aligned}
$$

By using the LKF (29) and similar arguments to the ones of Theorem 4.1, we obtain

$$
\dot{V}_{0}(t, z(t)) \leq-\zeta_{0}^{T}(t, z(t)) \bar{\Lambda} \zeta_{0}(t, z(t))+\alpha V_{0}(t, z(t))
$$

where

$$
\begin{aligned}
\zeta_{0}(t, z(t))= & {\left[z^{T}(t), \dot{z}^{T}(t), \dot{z}^{T}(t-h(t)), z^{T}(t-\tau(t)), g^{T}(z(t)),\right.} \\
& \left.g^{T}(z(t-\tau(t))),\left(\int_{-\infty}^{t} K(t-s) g(z(s)) d s\right)^{T}\right]^{T}
\end{aligned}
$$

and

$\bar{\Lambda}=\left[\begin{array}{ccccccc}\Lambda_{11} & \Lambda_{12} & \Lambda_{13} & -\Pi_{14} & -\Pi_{17} & -\Pi_{18} & -\Pi_{19} \\ * & -\Pi_{22} & -\Pi_{23} & 0 & 0 & -\Pi_{28} & -\Pi_{29} \\ * & * & -\Pi_{33} & 0 & 0 & -\Pi_{38} & -\Pi_{39} \\ * & * & * & -\Pi_{44} & 0 & -\Pi_{48} & 0 \\ * & * & * & * & -\Pi_{77} & 0 & 0 \\ * & * & * & * & * & -\Pi_{88} & 0 \\ * & * & * & * & * & * & -\Pi_{99}\end{array}\right]$

with

$$
\begin{aligned}
& \Lambda_{11}=P C+C P+U_{1} \Sigma_{1}+\alpha P, \quad \Lambda_{12}=Q_{1} C \\
& \Lambda_{13}=-P D-C Q_{2}^{T} D
\end{aligned}
$$

Clearly, if

$$
\bar{\Lambda}>0
$$

then we have

$$
\dot{V}_{0}(t, z(t)) \leq \alpha V_{0}(t, z(t)) .
$$

By using Schur complements, 30, can be expressed as

$$
c R-\Gamma>0
$$

where

$$
R=\left[\begin{array}{cccccc}
\check{\Lambda}_{22} & \check{\Lambda}_{23} & 0 & 0 & \check{\Lambda}_{28} & \check{\Lambda}_{29} \\
* & \check{\Lambda}_{33} & 0 & 0 & \check{\Lambda}_{38} & \check{\Lambda}_{39} \\
* & * & \check{\Lambda}_{44} & 0 & \check{\Lambda}_{48} & 0 \\
* & * & * & \check{\Lambda}_{77} & 0 & 0 \\
* & * & * & * & \check{\Lambda}_{88} & 0 \\
* & * & * & * & * & \check{\Lambda}_{99}
\end{array}\right]
$$

with

$$
\begin{aligned}
c>0, \quad \check{Q}_{i},>0, i=1,2,5,7, \quad \check{U}_{1}>0, \quad \check{U}_{2}>0, \\
Q_{1}=c \check{Q}_{1}, \quad Q_{2}=c \check{Q}_{2}, \quad Q_{5}=c \check{Q}_{5}, \quad Q_{6}=c \check{Q}_{6} \\
Q_{7}=c \check{Q}_{7}, \quad U_{1}=c \check{U}_{1}, \quad U_{2}=c \check{U}_{2}, \quad T_{11}=c \check{T}_{11}, \\
T_{12}=c \check{T}_{12}, \quad T_{22}=c \check{T}_{22}, \quad \check{\Lambda}_{22}=-\tau \check{T}_{22}-\check{Q}_{6}+\check{Q}_{1}+\check{Q}_{1}^{T}, \\
\check{\Lambda}_{23}=-\check{Q}_{1} D-\check{Q}_{2}^{T} D, \quad \check{\Lambda}_{28}=-\check{Q}_{1} \tilde{A}, \quad \check{\Lambda}_{29}=-\check{Q}_{1} B, \\
\check{\Lambda}_{33}=\check{Q}_{6}\left(1-h^{*}\right)+D^{T} \check{Q}_{2} D+D^{T} \check{Q}_{2}^{T} D, \quad \check{\Lambda}_{38}=-D^{T} \check{Q}_{2} \tilde{A}, \\
\check{\Lambda}_{39}=D^{T} \check{Q}_{2} B, \quad \check{\Lambda}_{44}=-\tau \check{T}_{11}+\check{T}_{12}+\check{T}_{12}^{T}+\check{U}_{2} \Sigma_{1}, \\
\check{\Lambda}_{48}=-\check{U}_{2} \Sigma_{2}, \quad \check{\Lambda}_{77}=-\check{Q}_{5} \kappa+\check{U}_{1}, \quad \check{\Lambda}_{88}=\check{U}_{2}, \quad \check{\Lambda}_{99}=\check{Q}_{5} .
\end{aligned}
$$


and

$$
\Gamma=\left[\begin{array}{c}
\Lambda_{12}^{T} \\
-\Pi_{13}^{T} \\
-\Pi_{14}^{T} \\
-\Pi_{17}^{T} \\
-\Pi_{18}^{T} \\
-\Pi_{19}^{T}
\end{array}\right]\left(\Lambda_{11}\right)^{-1}\left[\begin{array}{llllll}
\Lambda_{12} & -\Pi_{13} & -\Pi_{14} & -\Pi_{17} & -\Pi_{18} & -\Pi_{19}
\end{array}\right]
$$

Since $\Lambda_{11}>0$ then it leads to $\Gamma>0$ and consequently $R>$ $\frac{1}{c} \Lambda>0$ from condition (27). Therefore (31) is satisfied by taking $c>\frac{\lambda_{\max }(T)}{\lambda_{\max }(R)}$. Thus, condition 27) implies that $\dot{V}_{0}(t, z(t)) \leq$ $\alpha V_{0}(t, z(t))$ and consequently $V(t, z(t)) \leq e^{\alpha T} V(z(0))$.

Furthermore, we have

$$
\begin{aligned}
\|x(0)\|^{2} & \leq \frac{1}{\lambda_{\min }(P)} x^{T}(0) P x(0) \\
& \leq \frac{1}{\lambda_{\min }(P)} x^{T}(0) R^{\frac{1}{2}} R^{-\frac{1}{2}} P R^{-\frac{1}{2}} R^{\frac{1}{2}} x(0) \\
& \leq \frac{\lambda_{\max }(\bar{P})}{\lambda_{\min }(P)} x^{T}(0) R x(0)
\end{aligned}
$$

Therefore

$$
V(0) \leq \gamma c_{1}
$$

where

$$
\begin{aligned}
\gamma & =\frac{\lambda_{\max }(\bar{P})}{\lambda_{\min }(P)}\left[2 \lambda_{\max }(P)+\bar{h} \lambda_{\max }\left(Q_{6}\right)+\tau^{2} \lambda_{\max }\left(T_{22}\right)\right. \\
& \left.+\sum_{j=1}^{n} q_{j} \mathbf{k}_{j} \max _{j} M_{j}^{2} \int_{0}^{\infty} u k_{j}(u) \mathrm{d} u\right] .
\end{aligned}
$$

and

$$
x^{T}(t) R x(t) \leq \frac{1}{\lambda_{\min }(\bar{P})} x^{T}(t) P x(t) \leq \frac{1}{\lambda_{\min }(\bar{P})} V(t, x(t)) .
$$

So conditions 28) and (33) imply that $x^{T}(t) R x(t)<c_{2}$ which achieves the proof.

Remark 4.4. In [70], the problem of FTB for Markovian jumping NNs with time-varying delays is studied by using the reciprocally convex combination technique where a double integral term appears in the LKF. Very recently, the authors of [71] have used the same approach where a triple integral term is added in the LKF. However, these novel LKFs also lead to a greater complexity in terms of inequalities and variables to be calculated and this complexity can cause numerical problems with a large number of neurons $([33])$. In our article we use a well know LKF (8), but based on the Jensen's integral inequality the upper bound of the LKF is estimated more tightly and then the conservatism with respect to the delays is reduced (see Example 5.1.

\subsection{Finite time Boundedness stabilization}

In this section, sufficient conditions are given for solving the FTB-stabilization problem of a general class of NHOHNNs with time delay in the leakage term and mixed time delays of the form

$$
\left\{\begin{aligned}
\dot{x}_{i}(t) & =-c_{i} x_{i}(t-\sigma)+\sum_{j=1}^{n} a_{i j} f_{j}\left(x_{j}(t-\tau(t))\right) \\
& +\sum_{j=1}^{n} \sum_{k=1}^{n} T_{i j k} f_{k}\left(x_{k}(t-\tau(t))\right) f_{j}\left(x_{j}(t-\tau(t))\right) \\
& +\sum_{j=1}^{n} b_{i j} \int_{-\infty}^{t} k_{j}(t-s) f_{j}\left(x_{j}(s)\right) d s \\
& +\sum_{j=1}^{n} d_{i j} \dot{x}_{j}(t-h(t))+u, \quad t>0, i=1, \ldots, n \\
x(s) & =\phi(s), \quad s \in(-\infty, 0]
\end{aligned}\right.
$$

where $u$ is the control variable. The following state feedback control is considered

$$
u(x(t))=K_{2} x(t)
$$

where $K_{2}$ is to be determined. The corresponding closed-loop $z$-system is given by

$$
\left\{\begin{aligned}
\dot{z}(t) & =-C z(t-\sigma)+A g\left(z(t-\tau(t))+\Gamma^{T} T^{*} g(z(t-\tau(t)))\right. \\
& +B \int_{-\infty}^{t} K(t-s) g(z(s)) d s+D \dot{z}(t-h(t)) K_{2} z(t) \\
z(s) & =\phi(s)-x^{*}, \quad s \in(-\infty, 0]
\end{aligned}\right.
$$

We now introduce the definition of finite time bounded stabilization.

Definition 4.6. ([48]) The NNs described by System (35) is said to be FTB-stabilizable w.r.t. $\left(c_{1}, c_{2}, R, T\right)$ if there exists a controller $u(z(t))$ of the form (36) such that the corresponding closed-loop $z$-System (37) is FTB with respect to $\left(c_{1}, c_{2}, R, T\right)$.

We can now state the main result of this section.

Theorem 4.7. Under the assumptions and notations of Theorem 4.1. System (35) is FTB-stabilizable w.r.t. $\left(c_{1}, c_{2}, R, T\right)$ if the following conditions hold:

$$
\Psi=\left[\begin{array}{ccccccccc}
\Psi_{11} & \Psi_{12} & \Psi_{13} & 0 & \Pi_{15} & \Psi_{16} & \Pi_{17} & \Pi_{18} & \Pi_{19} \\
* & \Pi_{22} & \Pi_{23} & 0 & \Pi_{25} & 0 & 0 & \Pi_{28} & \Pi_{29} \\
* & * & \Pi_{33} & 0 & \Pi_{35} & \Pi_{36} & 0 & \Pi_{38} & \Pi_{39} \\
* & * & * & \Pi_{44} & 0 & 0 & 0 & \Pi_{48} & \Pi_{49} \\
* & * & * & * & \Pi_{55} & 0 & 0 & 0 & 0 \\
* & * & * & * & * & \Pi_{66} & 0 & \Pi_{68} & \Pi_{69} \\
* & * & * & * & * & * & \Pi_{77} & 0 & 0 \\
* & * & * & * & * & * & * & \Pi_{88} & 0 \\
* & * & * & * & * & * & * & * & \Pi_{99}
\end{array}\right]<0
$$

and

$$
\left[\begin{array}{cc}
c_{2} e^{-\alpha T} & \sqrt{c_{1}} \\
\sqrt{c_{1}} & \omega_{1}^{-1}
\end{array}\right]>0
$$

where

$$
\begin{aligned}
\tilde{C} & =C-K_{2}, \\
\Psi_{11} & =-P C-C P+P K_{2}+K_{2}^{T} P+Q_{3}+\sigma^{2} Q_{4}-U_{1} \Sigma_{1}-\alpha P, \\
\Psi_{12} & =-Q_{1} K_{2}, \quad \Psi_{13}=P D+D^{T} Q_{2} K_{2}, \quad \Psi_{16}=C P \tilde{C}+\alpha C P,
\end{aligned}
$$


ProOF. According to Definition 4.6 if we apply Theorem 4.1 to the closed-loop System (37) then we can easily obtain the result. The details of the proof is left to the reader.

When $\sigma=0$, Theorem 4.7 cannot be directly used to design a feedback control of the form of (36). To overcome this obstacle, the following proposition is established.

Proposition 4.8. Under assumptions $\left(H_{1}\right)-\left(H_{3}\right)$, System (35) without leakage delay is FTB-stabilizable w.r.t. $\left(c_{1}, c_{2}, T, R\right)$ if there exist a positive scalar $\alpha$, three $n \times n$ matrices $\tilde{Q}_{1}, \tilde{Q}_{2}, Y$, five $n \times n$ positive diagonal matrices $X, \tilde{U}_{1}, \tilde{U}_{2}, \tilde{Q}_{5}, Q_{6}$ and $a$ $2 n \times 2 n$ matrix

$$
\tilde{Q}_{7}=\left[\begin{array}{cc}
\tilde{T}_{11} & \tilde{T}_{12} \\
* & T_{22}
\end{array}\right]>0
$$

such that the following conditions hold:

$$
\Xi_{1}=\left[\begin{array}{ccccccc}
\Xi_{11} & \Xi_{12} & \Xi_{13} & \Xi_{14} & \Xi_{15} & \Xi_{16} & \Xi_{17} \\
* & \Xi_{22} & \Xi_{23} & 0 & 0 & \Xi_{26} & \Xi_{27} \\
* & * & \Xi_{33} & 0 & 0 & \Xi_{36} & \Xi_{37} \\
* & * & * & \Xi_{44} & 0 & \Xi_{46} & 0 \\
* & * & * & * & \Xi_{55} & 0 & 0 \\
* & * & * & * & * & \Xi_{66} & 0 \\
* & * & * & * & * & * & \Xi_{77}
\end{array}\right]<0
$$

and

$$
\left[\begin{array}{cc}
c_{2} e^{-\alpha T} & \sqrt{c_{1}} \\
\sqrt{c_{1}} & \bar{\omega}_{2}^{-1}
\end{array}\right]>0
$$

where

$$
\begin{aligned}
& \Xi_{11}=-C X-X C+Y+Y^{T}-\tilde{U}_{1} \Sigma_{1}-\alpha X, \\
& \Xi_{12}=X C+Y, \Xi_{13}=D+D^{T} C X+D^{T} Y, \\
& \Xi_{14}=\tilde{T}_{12}^{T}, \Xi_{15}=\tilde{U}_{1} \Sigma_{2}, \Xi_{16}=\tilde{A} X, \Xi_{17}=B X, \\
& \Xi_{22}=\tau T_{22}+Q_{6}-2 I, \Xi_{23}=X D+D^{T} X,, \\
& \Xi_{26}=X \tilde{A}, \Xi_{27}=X B, \Xi_{33}=-Q_{6}\left(1-h^{*}\right)-2 D^{T} D, \\
& \Xi_{36}=-X D^{T} \tilde{A}, \Xi_{37}=-X D^{T} B, \\
& \Xi_{44}=\tau \tilde{T}_{11}-\tilde{T}_{12}-\tilde{T}_{12}^{T}-\tilde{U}_{2} \Sigma_{1}, \Xi_{46}=\tilde{U}_{2} \Sigma_{2}, \\
& \Xi_{55}=\tilde{Q}_{5} \kappa-\tilde{U}_{1}, \Xi_{66}=-\tilde{U}_{2}, \Xi_{77}=-\tilde{Q}_{5} .
\end{aligned}
$$

and

$$
\begin{aligned}
\bar{\omega}_{2} & =\frac{1}{\lambda_{\min }(\bar{X})}\left[2 \lambda_{\max }\left(X^{-1}\right)+\bar{h} \lambda_{\max }\left(Q_{6}\right)\right. \\
& \left.+\tau^{2} \lambda_{\max }\left(T_{22}\right)+\sum_{j=1}^{n} \rho_{j}^{-1} \tilde{q}_{j} \rho_{j}^{-1} \mathbf{k}_{j} \max _{j} M_{j}^{2} \int_{0}^{\infty} u k_{j}(u) d u\right]
\end{aligned}
$$

with

$\tilde{Q}_{5}=\operatorname{diag}\left(\tilde{q}_{1}, \ldots, \tilde{q}_{n}\right), X=\operatorname{diag}\left(\rho_{1}, \ldots, \rho_{n}\right), \bar{X}=R^{\frac{-1}{2}} X^{-1} R^{\frac{-1}{2}}$.

ProOF. Let $Q_{1}=Q_{2}=I$. Now, we make some transformations for the above inequalities (40)-(41). Pre and post-multiplying (40) by $\operatorname{diag}\left(X^{-1}, I, I, X^{-1}, \ldots, X^{-1}\right)$ and letting

$$
\begin{aligned}
X & =P^{-1}, \quad Y=K X, \quad \tilde{Q}_{5}=P^{-1} Q_{5} P^{-1} \\
\tilde{U}_{1} & =P^{-1} U_{1} P^{-1}, \tilde{U}_{2}=P^{-1} U_{2} P^{-1}, \tilde{T}_{12}=P^{-1} T_{12} P^{-1} .
\end{aligned}
$$

we obtain $\Lambda>0$. That is, (40) implies Condition (27). Then, by replacing (42) in Condition (41) we obtain easily (28). Therefore, the conditions of Corollary 4.5 are obtained which achieves the proof.

Remark 4.5. Based on the inequality $S^{-1} \geq 2 \xi I-\xi^{2} S$ ( $S$ being a non-singular matrix), the following LMI conditions

$$
\begin{aligned}
& {\left[\begin{array}{cc}
-\lambda_{1} I & I \\
* & -X_{i}
\end{array}\right]<0, \quad X_{i}<\lambda_{2} I, \quad \tilde{Q}_{5}<2 \xi_{1} I-\xi_{1}^{2} \lambda_{3} I} \\
& {\left[\begin{array}{cc}
-\left(2 \xi_{3} I-\xi_{3}^{2} \lambda_{2}\right) \pi_{1} c_{2} e^{-\alpha T}+c_{1}\left[2 \lambda_{1}+\bar{h} \lambda_{6}+\tau^{2} \lambda_{7}\right] & \sqrt{c_{1} r_{3}} \lambda_{1} \\
* & -\lambda_{3} .
\end{array}\right]<0}
\end{aligned}
$$

where $\pi_{1}=\lambda_{\min }\left(R^{-1}\right), r_{3}=\sum_{j=1}^{n} \mathbf{k}_{j} \max _{j} M_{j}^{2} \int_{0}^{\infty} u k_{j}(u) d u, \xi_{1}, \xi_{3}$ are adjustable parameters and $\lambda_{i}, i=1,2,3,6,7$ are unknown positive scalars, ensure Condition (41) [48].

When $\sigma \neq 0$, the following proposition can be applied directly to design a feedback control which is able to FTB-stabilize the NHOHNNs.

Proposition 4.9. Under the assumptions $(H 1)-(H 2)-(H 3)$, if there exist a positive scalar $\alpha$, three positive symmetric definite matrices $P, Q_{3}, Q_{4}$, five $n \times n$ diagonal matrices $X, U_{1}>$ $0, U_{2}>0, Q_{5}>0, Q_{6}>0$, and $a 2 n \times 2 n$ matrix

$$
Q_{7}=\left[\begin{array}{cc}
T_{11} & T_{12} \\
* & T_{22}
\end{array}\right]>0
$$

such that the following conditions hold:

$$
\Theta=\left[\begin{array}{ccccccccc}
\Theta_{11} & X & \Pi_{13} & 0 & \Pi_{15} & \Theta_{16} & \Pi_{17} & \Pi_{18} & \Pi_{19} \\
* & \Theta_{22} & \Theta_{23} & 0 & \Theta_{25} & 0 & 0 & \Theta_{28} & \Theta_{29} \\
* & * & \Theta_{33} & 0 & 0 & \Pi_{36} & 0 & 0 & 0 \\
* & * & * & \Pi_{44} & 0 & 0 & 0 & \Pi_{48} & \Pi_{49} \\
* & * & * & * & \Pi_{55} & 0 & 0 & 0 & 0 \\
* & * & * & * & * & \Pi_{66} & 0 & \Pi_{68} & \Pi_{69} \\
* & * & * & * & * & * & \Pi_{77} & 0 & 0 \\
* & * & * & * & * & * & * & \Pi_{88} & 0 \\
* & * & * & * & * & * & * & * & \Pi_{99}
\end{array}\right]<0
$$

and

$$
\left[\begin{array}{cc}
c_{2} e^{-\alpha T} & \sqrt{c_{1}} \\
\sqrt{c_{1}} & \omega_{1}^{-1}
\end{array}\right]>0
$$

where

$\Theta_{11}=-P C-C P+Q_{3}+\sigma^{2} Q_{4}-U_{1} \Sigma_{1}-\alpha P+2 X, \Theta_{16}=C P C+\alpha C P-C X$, $\Theta_{22}=\tau T_{22}+Q_{6}-2 P, \Theta_{23}=P D, \Theta_{25}=-P C, \Theta_{28}=P \tilde{A}, \Theta_{29}=P B$, $\Theta_{33}=-Q_{6}\left(1-h^{*}\right)$

and other parameters are the same as in Theorem 4.1. then the feedback control $u_{2}(x(t))=P^{-1} X x(t)$ FTB-stabilize System (35) w.r.t. $\left(c_{1}, c_{2}, R, T\right)$.

Proof. Let $Q_{1}=P$ and $Q_{2}=0$. The proof of Proposition 4.9 is similar to the one of Theorem 4.7 so it is omitted here.

Remark 4.6. In the results presented in [47, 48, 42, 63, 64], the feedback control given for ensuring the FTB-stabilization of the NNs cannot be designed with a leakage delay. When $\sigma \neq 0$, the conditions established in these works are not LMI conditions which renders the control algorithm more complicated. 
To overcome these difficulties, the matrix gain should be of the following form $K=P^{-1} X$. In this case, it is possible to find LMI conditions ensuring the FTB-stabilization of NNs of the above mentioned works, even if there is a leakage delay.

\section{Numerical examples}

In this section, three numerical examples are presented to show the effectiveness of the results.

\subsection{Example 1}

Consider System (1) with $n=2$ and

$$
\begin{aligned}
f_{1}(s) & =f_{2}(s)=\tanh (s), \quad \tau(t)=0.2-0.1 \cos t, \\
h(t) & =\sigma=0.1, \quad k_{1}(s)=k_{2}(s)=e^{-s}, \quad J=(1,2)^{T},
\end{aligned}
$$

and parameters $C, A, T_{1}, T_{2}, B$ and $D$ given as follows:

$$
\begin{aligned}
C & =\left[\begin{array}{ll}
4 & 0 \\
0 & 4
\end{array}\right], A=\left[\begin{array}{cc}
0.1 & 0.18 \\
-0.8 & -0.93
\end{array}\right], T_{1}=\left[\begin{array}{cc}
0.2 & 0.07 \\
0.03 & 0.01
\end{array}\right], \\
T_{2} & =\left[\begin{array}{cc}
0.1 & 0.05 \\
0.04 & 0.02
\end{array}\right], B=\left[\begin{array}{cc}
0.7 & -0.2 \\
-0.2 & 0.5
\end{array}\right], D=\left[\begin{array}{cc}
0.1 & 0 \\
0 & 0.1
\end{array}\right] .
\end{aligned}
$$

It leads to $\tau=0.3, h^{*}=0, M_{j}^{-}=0, M_{j}^{+}=1, k_{j}=1$ for $j=1,2$. Note that

$$
C-A^{+} M-B^{+} K^{+} M=\left[\begin{array}{cc}
3.2 & -0.38 \\
-1 & 2.57
\end{array}\right]
$$

is a M-matrix. By using Theorem 3.1, we know that System (1) has an equilibrium point.

For $c_{1}=0.35, T=5$ and $R=I$, solving (6)-(7) with the Matlab LMI toolbox [72] with $\alpha=0.02$ leads to the following solutions

$$
\begin{aligned}
P & =\left[\begin{array}{cc}
128.0037 & 19.7975 \\
19.7975 & 62.5951
\end{array}\right], Q_{1}=\left[\begin{array}{cc}
10.08 & 3.3704 \\
3.3704 & 6.3560
\end{array}\right] \\
Q_{2} & =\left[\begin{array}{ll}
16.7695 & 1.7013 \\
1.7013 & 7.4374
\end{array}\right], Q_{3}=\left[\begin{array}{cc}
220.9824 & 57.9710 \\
57.9710 & 131.3136
\end{array}\right] \\
Q_{4} & =\left[\begin{array}{ll}
7.3255 & 1.2523 \\
1.2523 & 5.1806
\end{array}\right], Q_{5}=\left[\begin{array}{cc}
110.8764 & 0 \\
0 & 65.8265
\end{array}\right] \\
Q_{6} & =\left[\begin{array}{cc}
2.0464 & 0 \\
0 & 0.8882
\end{array}\right], U_{1}=\left[\begin{array}{cc}
232.5544 & 0 \\
0 & 156.5080
\end{array}\right] \\
U_{2} & =\left[\begin{array}{cc}
65.8755 & 0 \\
0 & 58.9563
\end{array}\right], T_{11}=\left[\begin{array}{cc}
112.1715 & 54.9582 \\
54.9582 & 85.9251
\end{array}\right] \\
T_{12} & =\left[\begin{array}{ll}
45.4573 & 20.3443 \\
20.3443 & 31.9253
\end{array}\right], T_{22}=\left[\begin{array}{cc}
19.7736 & 8.8448 \\
8.8448 & 13.8120
\end{array}\right]
\end{aligned}
$$

The minimum value of $c_{2}$ satisfies $\min c_{2}>1.8157$ for $\alpha=$ 0.02 . Theorem 4.1 leads to the FTB of the considered system w.r.t. $(0.35,3, I, 5)$. Time history of $x^{T}(t) x(t)$ is illustrated on Fig 1 .

Moreover, we see on Figure 2 that the considered system is not FTB w.r.t. $(0.35,0.9, I, 100)$.

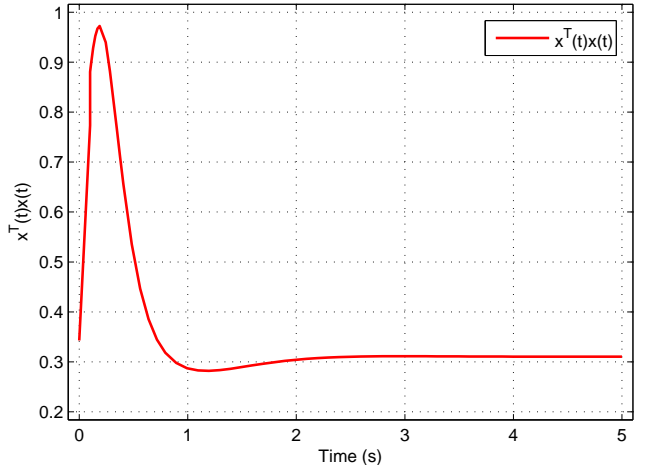

Figure 1: Time history of $x^{T}(t) x(t)$ with initial condition $(0.305,0.5)^{T}$

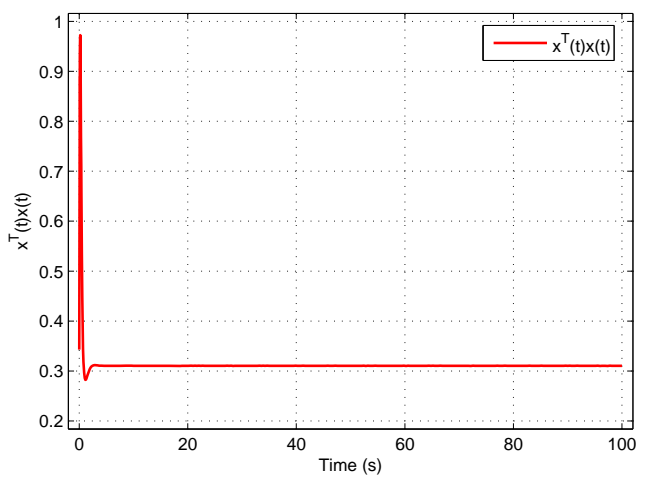

Figure 2: Time history of $x^{T}(t) x(t)$ with initial condition $(0.305,0.5)^{T}$ for $T=100$

Now, we fix $\alpha=0.02$ and plot on Figure 3 the different values of the maximum of $c_{1}$ with respect to the parameters $T$ and $c_{2}$ for having the FTB.

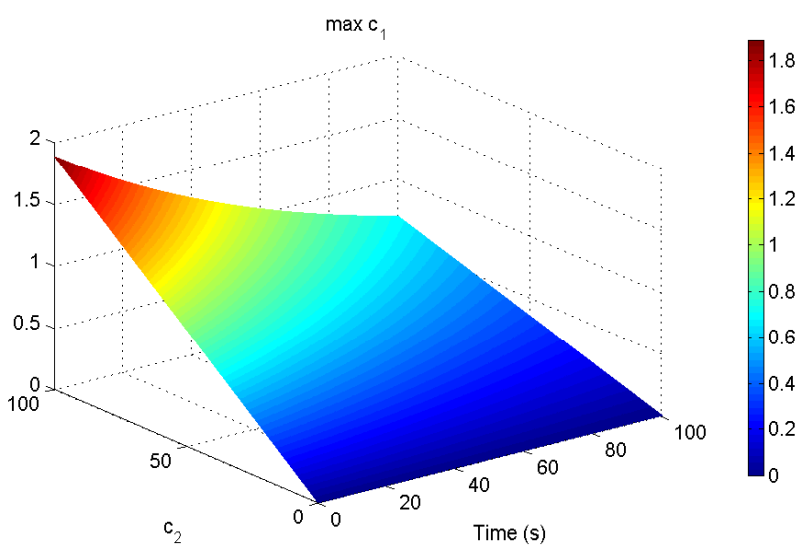

Figure 3: $\max c_{1}$ with respect to $\left(T, c_{2}\right)$

Finally for $\alpha=0.01$, Figure 4 shows the minimum of $c_{2}$ with respect to $\left(T, c_{1}\right)$ for having the FTB. 


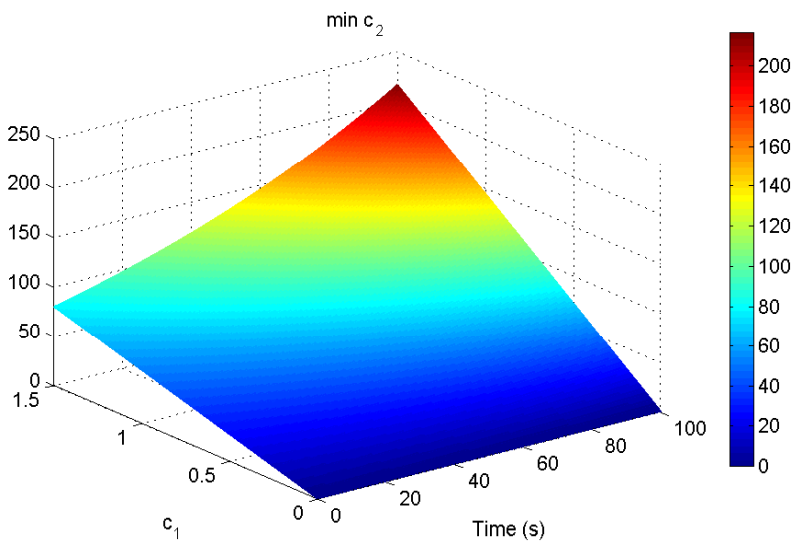

Figure 4: $\min c_{2}$ with respect to $\left(T, c_{1}\right)$

\begin{tabular}{|l|c|}
\hline \multicolumn{2}{|c|}{$h^{*}=0$ and $\sigma=0$} \\
\hline \hline Methods & $\tau_{\max }$ \\
\hline$[73]$ & 0.87 \\
\hline$[15]$ & 0.3527 \\
\hline$[75]$ & 0.4 \\
\hline Corollary 4.5 & 1.123 \\
\hline
\end{tabular}

Table 1: The maximum allowable bounds $\tau_{\max }$ for $h^{*}=0$ and $\sigma=0$

When $\sigma=0$, the upper bound of the delay $\tau$ for which LMIs (6) of Theorem 4.1 remain feasible is $\tau_{\max }=1.088$. However, we obtain $\tau_{\max }=1.123$ if we use the simplified criterion given in Corollary 4.5 .

Remark 5.1. The stability criterion given in [15, 73, 74, 75] fails for small delays ( $\tau_{\max } \leq 0.87$ ) and becomes infeasible when a leakage delay $\sigma$ extends beyond 0.15 . The method proposed in our work overcomes these difficulties by proving sufficient conditions that are able to ensure the FTB and even the asymptotic stability for a delay larger than the one given in [15, 73, 75]. Table 1 and Table 2 compare the maximum allowable bounds $\tau_{\max }$ of $\tau(t)$ derived from Theorem 4.1. Corollary 4.5 and $[15,73,75,74]$.

Remark 5.2. The method used in our article improves and extends the results given in [15, 74] by reducing the conservatism with respect to the delays and by simplifying the calculus simultaneously. This improvement is illustrated in Tables 3 and 5 by presenting a comparison of the computational load. Noted that FWM and DV stand respectively for the number of

\begin{tabular}{|l|c|c|}
\hline \multicolumn{3}{|c|}{$h^{*}=0$} \\
\hline \hline Methods & $\tau_{\max }$ for $\sigma=0.15$ & $\tau_{\max }$ for $\sigma=0.2$ \\
\hline$[74]$ & Infeasible & Infeasible \\
\hline$[$ Theorem 4.1 & 0.245 & 0.095 \\
\hline
\end{tabular}

Table 2: The maximum allowable bounds of $\tau_{\max }$ for $h^{*}=0$

\begin{tabular}{|l|c|c|c|}
\hline \multicolumn{4}{|c|}{$\sigma=0$} \\
\hline \hline Methods & FWM & DV & $\tau_{\max }$ \\
\hline$[15]$ & 2 & $4 n^{2}+4 n$ & 0.3527 \\
\hline$[$ Corollary 4.5 & 2 & $3 n^{2}+4 n+1$ & 1.123 \\
\hline
\end{tabular}

Table 3: A comparison of computational load when $\sigma=0$

\begin{tabular}{|l|c|c|c|}
\hline \multicolumn{4}{|c|}{$\sigma=0.15$} \\
\hline \hline Methods & FWM & DV & $\tau_{\max }$ \\
\hline$[74]$ & 2 & $28 n^{2}+16 n$ & Infeasible \\
\hline$[$ Theorem 4.1] & 2 & $8.5 n^{2}+4 n+1$ & 0.245 \\
\hline
\end{tabular}

Table 4: A comparison of computational load when $\sigma=0.15$

free-weight matrices and the number of decision variables used.

\subsection{Example 2}

Consider System (1) with $n=2$,

$$
C=\left[\begin{array}{cc}
1 & 0 \\
0 & 0.2
\end{array}\right]
$$

and other parameters similar to Subsection 5.1. By setting $c_{1}=$ $6.5, T=5$ and $R=I$, we have that System (1) is FTB w.r.t. $(6.5,1500, I, 5)$ but is not FTB w.r.t. $(6.5,1500, I, 10)$. Moreover, we have $\left|x_{i}\right| \rightarrow+\infty, i=1,2$ when $t \rightarrow+\infty$ as shown if Figure 5 which proves that this system is not asymptotically stable.

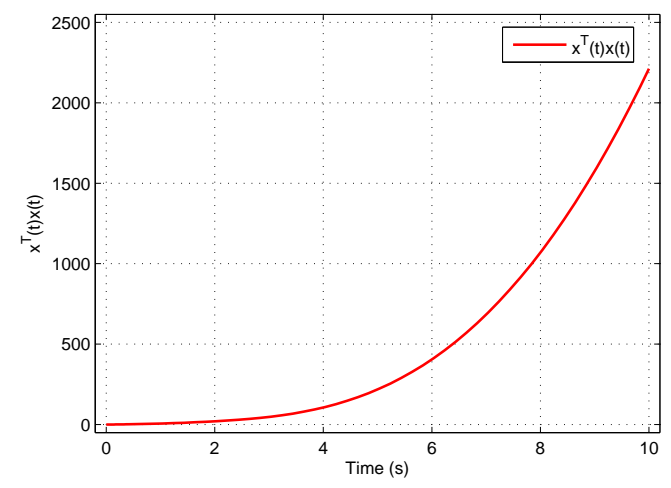

Figure 5: The history of $x^{T}(t) x(t)$ for System (1) in Exemple 2 with initial condition $(0.1,0.1)^{T}$

\begin{tabular}{|l|c|c|c|}
\hline \multicolumn{4}{|c|}{$\sigma=0.2$} \\
\hline \hline Methods & FWM & DV & $\tau_{\max }$ \\
\hline$[74]$ & 2 & $28 n^{2}+16 n$ & Infeasible \\
\hline$[$ Theorem 4.1] & 2 & $8.5 n^{2}+4 n+1$ & 0.095 \\
\hline
\end{tabular}

Table 5: The maximum allowable bounds of $\tau(t)$ when $\sigma=0.2$ 
So, we have shown that FTB and Lyapunov asymptotic stability are two independent concepts.

Now we consider the controlled System (35) with the above numerical values. In order to FTB-stabilize System (35), we consider the feedback control according to the method proposed in Theorem 4.7. The feedback control is given by $u(x(t))=$ $K_{1} x(t)$, with $K_{1}=\left[\begin{array}{cc}-0.1 & 0 \\ 0 & 0.042\end{array}\right]$. From Theorem 4.7, we deduce that System 35 is FTB stabilizable w.r.t. $(6.5,1500, I, 10)$. Figure 6 shows the history of $x^{T}(t) x(t)$.

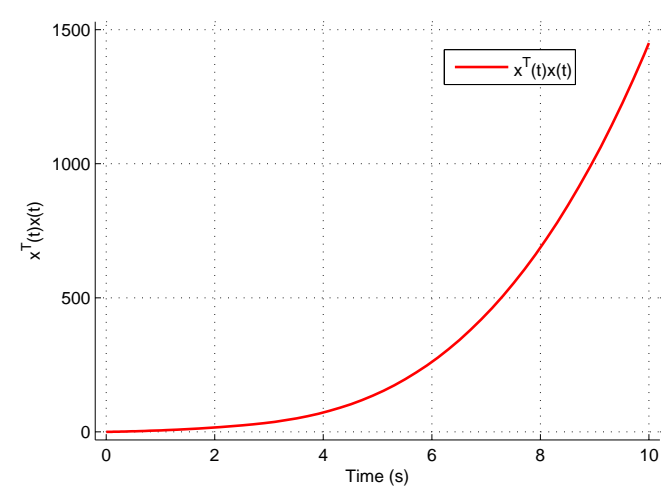

Figure 6: The history of $x^{T}(t) x(t)$ for System 35 with initial condition $(0.1,0.1)^{T}$ under controller 36 with $K=K_{1}$

The state feedback control $u_{2}(x(t))=K_{2} x(t)$ with

$$
K_{2}=\left[\begin{array}{cc}
-0.1 & 0 \\
0 & -0.9
\end{array}\right]
$$

FTB-stabilize the system but it also asymptotically stabilize the system if we consider an infinite time evolution. The history of $x^{T}(t) x(t)$ is then illustrated in Figure 7 .

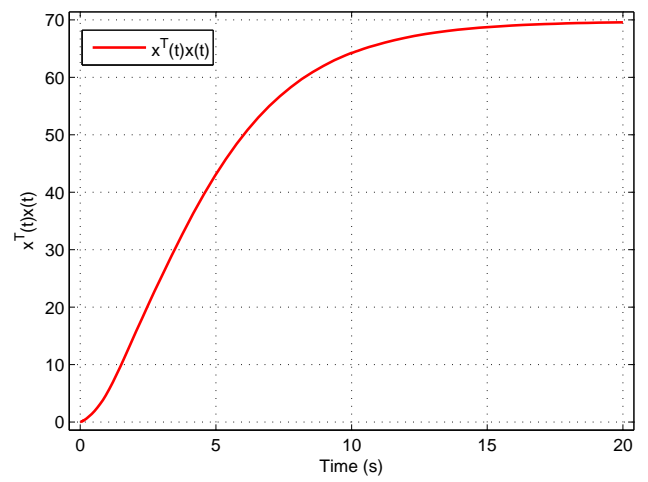

Figure 7: The history of $x^{T}(t) x(t)$ for System 37 with initial condition $(0.1,0.1)^{T}$ under controller [36 with $K=K_{2}$

\subsection{Example 3}

Consider System (1) with $n=2$,

$$
C=\left[\begin{array}{cc}
0.01 & 0 \\
0 & 0.01
\end{array}\right]
$$

and other parameters similar to Subsection 5.1. From condition (44), we have that $\min c_{2}=11.6515$. We choose $c_{1}=0.35$, $T=10$ and $c_{2}=12$ and we see on Figure 8 that the open-loop System (1) is not FTB w.r.t. $\left(c_{1}, c_{2}, I, T\right)$.

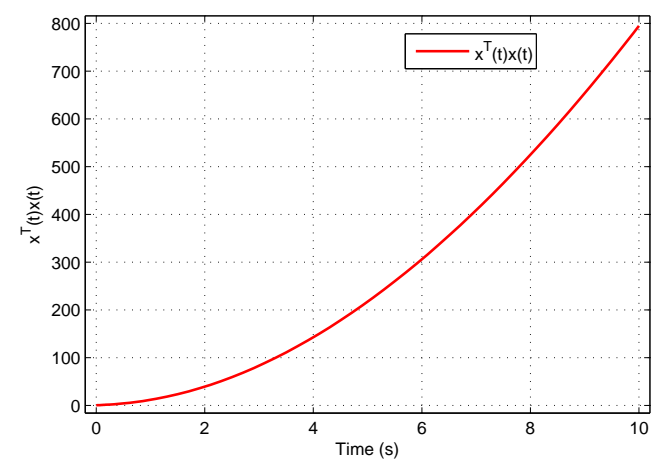

Figure 8: The history of $x^{T}(t) x(t)$ for System 37] without controller 36

In order to FTB stabilize System (35) with the above numerical values, we build the following control $U(x(t))=P^{-1} X x(t)$ according to the strategy given in Subsection 4.2 Proposition 4.9 implies that the closed-loop System (37) is FTB-stabilizable w.r.t. $\left(c_{1}, c_{2}, T, I\right)$ for $\alpha=0.02$, with the following solutions

$$
\begin{aligned}
P & =\left[\begin{array}{cc}
6.5871 & 0 \\
0 & 4.3602
\end{array}\right], Q_{3}=\left[\begin{array}{cc}
0.3573 & -0.1380 \\
-0.1380 & 0.2511
\end{array}\right] \\
Q_{4} & =\left[\begin{array}{cc}
4.8974 & -0.5024 \\
-0.5024 & 4.5153
\end{array}\right], Q_{5}=\left[\begin{array}{cc}
6.1356 & 0 \\
0 & 3.5997
\end{array}\right] \\
Q_{6} & =\left[\begin{array}{cc}
0.5134 & 0 \\
0 & 0.3434
\end{array}\right], U_{1}=\left[\begin{array}{cc}
12.0818 & 0 \\
0 & 7.2642
\end{array}\right] \\
U_{2} & =\left[\begin{array}{cc}
11.2816 & 0 \\
0 & 8.7200
\end{array}\right], T_{11}=\left[\begin{array}{cc}
4.6319 & 0.5660 \\
0.5660 & 3.5918
\end{array}\right] \\
T_{12} & =\left[\begin{array}{cc}
4.0306 & 1.0331 \\
1.0331 & 3.1048
\end{array}\right], T_{22}=\left[\begin{array}{ll}
3.7924 & 1.3756 \\
1.3756 & 2.8913
\end{array}\right] \\
X & =\left[\begin{array}{cc}
-14.7082 & 0 \\
0 & -5.5260
\end{array}\right]
\end{aligned}
$$

and the controller $u(x(t))=P^{-1} X x(t)$ with

$$
P^{-1} X=\left[\begin{array}{cc}
-2.2329 & 0 \\
0 & -1.2674
\end{array}\right]
$$

The history of $x^{T}(t) R x(t)$ with the previous controller $u(x(t))=$ $P^{-1} X x(t)$ is illustrated on Figure 9 


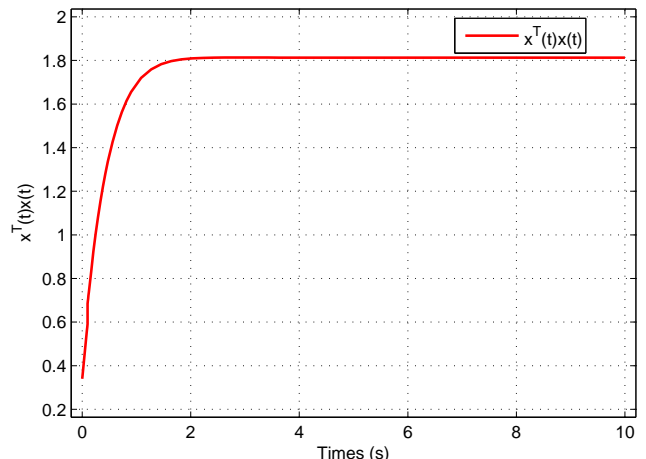

Figure 9: The history of $x^{T}(t) x(t)$ for System 37 under controller $P^{-1} X x(t)$.

Now, if the time-varying delay $\tau(t)$ defined by the non-differentiable function is as follows:

$$
\tau(t)= \begin{cases}0.3-0.1 \sin t & \text { if } \quad t \in \mathfrak{I}=\cup_{k \geq 0}[2 k \pi,(2 k+1) \pi] ; \\ 0 & \text { if } \quad t \in \mathbb{R}^{+} \backslash \mathfrak{I} .\end{cases}
$$

System (35) stays FTB-stabilizable w.r.t. $\left(c_{1}, c_{2}, T, I\right)$ which is illustrated in Figure 10

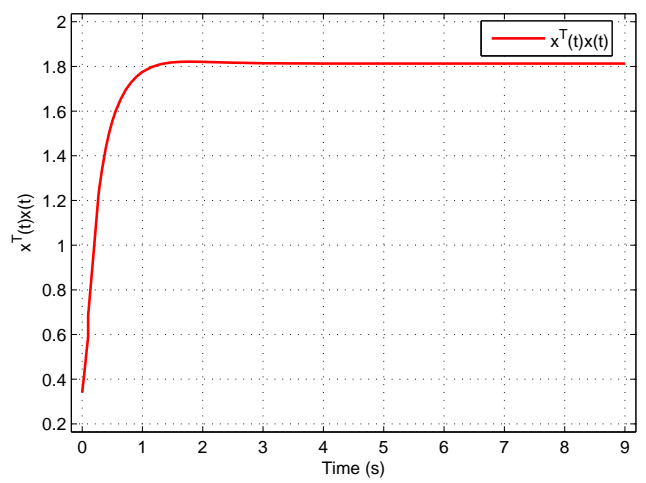

Figure 10: The history of $x^{T}(t) x(t)$ for System 37 under time-varying delay 45 .

Remark 5.3. It should be pointed out that the results in [47, 48, 42, 63, 64] fail for System (1) with the time-varying delay 45] even without leakage delay because (45) is not differentiable. This proves the advantage of the proposed approach in our article.

\section{Conclusion}

This article deals with the problem of finite time boundedness and finite time boundedness stabilization of a general class of neutral high-order Hopfield neural networks with time delay in the leakage term and mixed time delays. By using the topological degree theory, sufficient conditions are given to prove the existence of equilibrium points. The Lyapunov-Krasovskii functional method and the LMIs technique are used to establish some sufficient conditions which ensure the finite time boundedness and finite time boundedness stabilization of the class of systems considered in our article. Finally, numerical examples are presented to show the effectiveness and the interest of our proposed results.

\section{AppendixA. The calculus of $\dot{V}(t)$}

System (1) has an equivalent form given by

$$
\left\{\begin{array}{l}
\frac{d}{d t}\left[z(t)-C \int_{t-\sigma}^{t} z(u) d u\right]=-C z(t)+\left(A+\Gamma^{T} T^{*}\right) g(z(t-\tau(t))) \\
+B \int_{-\infty}^{t} K(t-s) g(z(s)) d s+D \dot{z}(t-h(t)) \\
z(s)=\phi(s)-x^{*}
\end{array}\right.
$$

Moreover, we have

$$
\begin{aligned}
\dot{V}_{1}(t, z(t)) & =2\left[z(t)-C \int_{t-\sigma}^{t} z(u) d u\right]^{T} P\left[-C z(t)+\left(A+\Gamma^{T} T^{*}\right) g(z(t-(\tau(t)))\right. \\
& \left.+D \dot{z}(t-h(t))+B \int_{-\infty}^{t} K(t-s) g(z(s)) d s\right] \\
& =-2 z^{T}(t) P C z(t)+2 z^{T}(t) P\left(A+\Gamma^{T} T^{*}\right) g(z(t-\tau(t))) \\
& +2 z^{T}(t) P B \int_{-\infty}^{t} K(t-s) g(z(s)) d s+2 z^{T}(t) P D \dot{z}(t-h(t)) \\
& +2 z^{T}(t) C P C \int_{t-\sigma}^{t} z(u) d u-2\left[\int_{t-\sigma}^{t} z(u) d u\right]^{T} C P D \dot{z}(t-h(t)) \\
& -2\left[\int_{t-\sigma}^{t} z(u) d u\right]^{T} C P\left(A+\Gamma^{T} T^{*}\right) g(z(t-(\tau(t))) \\
& -2\left[\int_{t-\sigma}^{t} z(u) d u\right]^{T} C P B \int_{-\infty}^{t} K(t-s) g(z(s)) d s
\end{aligned}
$$

and

$$
\begin{aligned}
\dot{V}_{2}(t, z(t)) & =z^{T}(t) Q_{3} z(t)-z^{T}(t-\sigma) Q_{3} z(t-\sigma)+\dot{z}^{T}(t) Q_{6} \dot{z}(t) \\
& -\dot{z}^{T}(t-h(t)) Q_{6} \dot{z}(t-h(t))(1-\dot{h}(t)) \\
& \leq z^{T}(t) Q_{3} z(t)-z^{T}(t-\sigma) Q_{3} z(t-\sigma)+\dot{z}^{T}(t) Q_{6} \dot{z}(t) \\
& -\dot{z}^{T}(t-h(t)) Q_{6} \dot{z}(t-h(t))\left(1-h^{*}\right)
\end{aligned}
$$

It follows from Lemma 2.5 that

$$
\begin{aligned}
\dot{V}_{3}(t, z(t)) & =\sigma^{2} z^{T}(t) Q_{4} z(t)-\sigma \int_{t-\sigma}^{t} z^{T}(u) Q_{4} z(u) d u \\
& \leq \sigma^{2} z^{T}(t) Q_{4} z(t)-\left[\int_{t-\sigma}^{t} z(u) d u\right]^{T} Q_{4}\left[\int_{t-\sigma}^{t} z(u) d u\right]
\end{aligned}
$$

and

$$
\begin{aligned}
\dot{V}_{4}(t, z(t)) & =\int_{t-\tau(t)}^{t}\left[\begin{array}{c}
g(z(t-\tau(t))) \\
\dot{z}(s)
\end{array}\right]^{T}\left[\begin{array}{cc}
T_{11} & T_{12} \\
* & T_{22}
\end{array}\right]\left[\begin{array}{c}
g(z(t-\tau(t))) \\
\dot{z}(s)
\end{array}\right] d s \\
& =\tau(t) z^{T}(t-\tau(t)) T_{11} z(t-\tau(t))+2 z^{T}(t) T_{12}^{T} z(t-\tau(t)) \\
& -2 z^{T}(t-\tau(t)) T_{12}^{T} z(t-\tau(t))+\int_{t-\tau(t)}^{t} \dot{z}^{T}(s) T_{22} \dot{z}(s) d s \\
& \left.\leq z^{T}(t-\tau(t))\left[\tau T_{11}-2 T_{12}^{T}\right] z(t-\tau(t))\right] \\
& +2 z^{T}(t) T_{12}^{T} z(t-\tau(t))+\int_{t-\tau}^{t} \dot{z}^{T}(s) T_{22} \dot{z}(s) d s
\end{aligned}
$$


and

$$
\begin{aligned}
\dot{V}_{5}(t, z(t)) & =\tau \dot{z}^{T}(t) T_{22} \dot{z}(t)-\int_{-\tau}^{0} \dot{z}^{T}(t+u) T_{22} \dot{z}(t+u) d u \\
& =\tau \dot{z}^{T}(t) T_{22} \dot{z}(t)-\int_{t-\tau}^{t} \dot{z}^{T}(s) T_{22} \dot{z}(s) d s
\end{aligned}
$$

and

$$
\begin{aligned}
\dot{V}_{6}(t, z(t)) & =\sum_{j=1}^{n} q_{j} k_{j} \int_{0}^{\infty} k_{j}(u)\left(g_{j}^{2}\left(z_{j}(t)\right)-g_{j}^{2}\left(z_{j}(t-u)\right)\right) d u \\
& \leq g^{T}(z(t)) Q_{5} \kappa g(z(t))-\sum_{j=1}^{n} q_{j} \int_{0}^{\infty} \kappa_{j}(u) d u \\
& \times \int_{0}^{\infty} \kappa_{j}(u) g_{j}^{2}\left(z_{j}(t-u)\right) d u \\
& \leq g^{T}(z(t)) Q_{5} \kappa g(z(t))-\sum_{j=1}^{n} q_{j}\left(\int_{0}^{\infty} k_{j}(u) g_{j}\left(z_{j}(t-u)\right) d u\right)^{2} \\
& \leq g^{T}(z(t)) Q_{5} \kappa g(z(t)) \\
& -\left(\int_{-\infty}^{t} K(t-s) g(z(s)) d s\right)^{T} Q_{5}\left(\int_{-\infty}^{t} K(t-s) g(z(s)) d s\right)
\end{aligned}
$$

In addition, we note that

$$
\begin{aligned}
0 & =2 \dot{z}^{T}(t) Q_{1}(-\dot{z}(t)+\dot{z}(t)) \\
& =-2 \dot{z}^{T}(t) Q_{1} \dot{z}(t)-2 \dot{z}^{T}(t) Q_{1} C z(t-\sigma)+2 \dot{z}^{T}(t) Q_{1}\left(A+\Gamma^{T} T^{*}\right) g(z(t-\tau(t))) \\
& +2 \dot{z}^{T}(t) Q_{1} D \dot{z}(t-h(t))+2 \dot{z}^{T}(t) Q_{1} B \int_{-\infty}^{t} K(t-s) g(z(s)) d s
\end{aligned}
$$

and

$$
\begin{aligned}
0 & =2 \dot{z}^{T}(t-h(t)) D^{T} Q_{2}(-D \dot{z}(t-h(t))+D \dot{z}(t-h(t))) \\
& =2 \dot{z}^{T}(t-h(t)) D^{T} Q_{2}(-D \dot{z}(t-h(t))+\dot{z}(t)+C z(t-\sigma) \\
& -\left(A+\Gamma^{T} T^{*}\right) g\left(z(t-\tau(t))-B \int_{-\infty}^{t} K(t-s) g(z(s)) d s\right) \\
& =-2 \dot{z}^{T}(t-h(t)) D^{T} Q_{2} D \dot{z}(t-h(t))+2 \dot{z}^{T}(t-h(t)) D^{T} Q_{2} D \dot{z}(t) \\
& +2 \dot{z}^{T}(t-h(t)) D^{T} Q_{2} C z(t-\sigma) \\
& -2 \dot{z}^{T}(t-h(t)) D^{T} Q_{2}\left(A+\Gamma^{T} T^{*}\right) g(z(t-\tau(t))) \\
& -2 \dot{z}^{T}(t-h(t)) D^{T} Q_{2} B \int_{-\infty}^{t} K(t-s) g(z(s)) d s
\end{aligned}
$$

By using $(H 1)$, the following inequality holds

$$
\begin{aligned}
& 0 \leq\left[\begin{array}{c}
z(t) \\
g(z(t))
\end{array}\right]^{T}\left[\begin{array}{cc}
-U_{1} \Sigma_{1} & U_{1} \Sigma_{2} \\
* & -U_{1}
\end{array}\right]\left[\begin{array}{c}
z(t) \\
g(z(t))
\end{array}\right] \\
& +\left[\begin{array}{c}
z(t-\tau(t)) \\
g(z(t-\tau(t)))
\end{array}\right]^{T}\left[\begin{array}{cc}
-U_{2} \Sigma_{1} & U_{2} \Sigma_{2} \\
* & -U_{2}
\end{array}\right]\left[\begin{array}{c}
z(t-\tau(t)) \\
g(z(t-\tau(t)))
\end{array}\right]
\end{aligned}
$$

for any $n \times n$ diagonal matrices $U_{1}>0, U_{2}>0$. By using A.1)-A.9, we finally get

$$
\dot{V}(t, z(t)) \leq \zeta^{T}(t, z(t)) \bar{\Xi} \zeta(t, z(t)) .
$$

\section{References}

[1] C. M. Bishop, Neural networks for pattern recognition, Oxford University Press, 1995.

[2] C. Aouiti, A. M. Alimi, A. Maalej, A genetic-designed beta basis function neural network for multi-variable functions approximation, Systems Analysis Modelling Simulation 42 (7) (2002) 975-1009.
[3] C. Aouiti, A. M. Alimi, F. Karray, A. Maalej, The design of beta basis function neural network and beta fuzzy systems by a hierarchical genetic algorithm, Fuzzy Sets and Systems 154 (2) (2005) 251-274.

[4] C. Aouiti, M. S. M'hamdi, F. Chérif, New results for impulsive recurrent neural networks with time-varying coefficients and mixed delays, Neural Processing Letter doi:10.1007/s11063-017-9601-y

[5] C. Aouiti, Oscillation of impulsive neutral delay generalized high-order Hopfield neural networks, Neural Computing and Applicationsdoi:10. 1007/s00521-016-2558-3

[6] C. Aouiti, M. S. M'hamdi, A. Touati, Pseudo almost automorphic solutions of recurrent neural networks with time-varying coefficients and mixed delays, Neural Processing Letters 45 (1) (2017) 121-140.

[7] H. Chen, S. Zhong, J. Shao, Exponential stability criterion for interval neural networks with discrete and distributed delays, Applied Mathematics and Computation 250 (2015) 121-130.

[8] H. Huang, Q. Du, X. Kang, Global exponential stability of neutral highorder stochastic Hopfield neural networks with Markovian jump parameters and mixed time delays, ISA Transactions 52 (6) (2013) 759-767.

[9] B. Liu, Almost periodic solutions for Hopfield neural networks with continuously distributed delays, Mathematics and Computers in Simulation 73 (5) (2007) 327-335.

[10] W. Peng, Q. Wu, Z. Zhang, LMI-based global exponential stability of equilibrium point for neutral delayed BAM neural networks with delays in leakage terms via new inequality technique, Neurocomputing 199 (2016) 103-113.

[11] Z. Wang, Y. Liu, X. Liu, On global asymptotic stability of neural networks with discrete and distributed delays, Physics Letters A 345 (4) (2005) 299-308.

[12] H. Huang, J. Cao, J. Wang, Global exponential stability and periodic solutions of recurrent neural networks with delays, Physics Letters A 298 (5) (2002) 393-404.

[13] B. Ammar, F. Chérif, A. M. Alimi, Existence and uniqueness of pseudo almost-periodic solutions of recurrent neural networks with time-varying coefficients and mixed delays, IEEE Transactions on Neural Networks and Learning Systems 23 (1) (2012) 109-118.

[14] K. Gopalsamy, Leakage delays in BAM, Journal of Mathematical Analysis and Applications 325 (2) (2007) 1117-1132.

[15] X. Li, J. Cao, Delay-dependent stability of neural networks of neutral type with time delay in the leakage term, Nonlinearity 23 (7) (2010) 17091726.

[16] Y. Li, Z. Zeng, S. Wen, Asymptotic stability analysis on nonlinear systems with leakage delay, Journal of The Franklin Institute 353 (3) (2016) 757 779.

[17] S. Peng, Global attractive periodic solutions of BAM neural networks with continuously distributed delays in the leakage terms, Nonlinear Analysis: Real World Applications 11 (3) (2010) 2141-2151.

[18] C. Aouiti, M. S. M'hamdi, J. Cao, A. Alsaedi, Piecewise pseudo almost periodic solution for impulsive generalised high-order Hopfield neural networks with leakage delays, Neural Processing Letters (2016) 1-34.

[19] P. Balasubramaniam, M. Kalpana, R. Rakkiyappan, Global asymptotic stability of BAM fuzzy cellular neural networks with time delay in the leakage term, discrete and unbounded distributed delays, Mathematical and Computer Modelling 53 (56) (2011) 839 - 853.

[20] S. Lakshmanan, J. H. Park, T. H. Lee, H. Jung, R. Rakkiyappan, Stability criteria for BAM neural networks with leakage delays and probabilistic time-varying delays, Applied Mathematics and Computation 219 (17) (2013) 9408 - 9423.

[21] X. Li, X. Fu, P. Balasubramaniam, R. Rakkiyappan, Existence, uniqueness and stability analysis of recurrent neural networks with time delay in the leakage term under impulsive perturbations, Nonlinear Analysis: Real World Applications 11 (5) (2010) 4092 - 4108.

[22] X. Li, R. Rakkiyappan, P. Balasubramaniam, Existence and global stability analysis of equilibrium of fuzzy cellular neural networks with time delay in the leakage term under impulsive perturbations, Journal of The Franklin Institute 348 (2) (2011) 135 - 155.

[23] Z. Wang, G. Wei, G. Feng, Reliable $H_{\infty}$ control for discrete-time piecewise linear systems with infinite distributed delays, Automatica 45 (12) (2009) 2991-2994.

[24] J.-e. Feng, S. Xu, Y. Zou, Delay-dependent stability of neutral type neural networks with distributed delays, Neurocomputing 72 (10) (2009) 25762580 . 
[25] X. Li, Global robust stability for stochastic interval neural networks with continuously distributed delays of neutral type, Applied Mathematics and Computation 215 (12) (2010) 4370-4384.

[26] C. Aouiti, Neutral impulsive shunting inhibitory cellular neural networks with time-varying coefficients and leakage delays, Cognitive Neurodynamics 10 (6) (2016) 573-591.

[27] H. Huang, G. Feng, State estimation of recurrent neural networks with time-varying delay: A novel delay partition approach, Neurocomputing 74 (5) (2011) $792-796$.

[28] S. Lakshmanan, K. Mathiyalagan, J. H. Park, R. Sakthivel, F. A. Rihan, Delay-dependent state estimation of neural networks with mixed timevarying delays, Neurocomputing 129 (2014) 392 - 400.

[29] X. Li, H. Gao, A new model transformation of discrete-time systems with time-varying delay and its application to stability analysis, IEEE Transactions on Automatic Control 56 (9) (2011) 2172-2178.

[30] T. Li, X. Yang, P. Yang, S. Fei, New delay-variation-dependent stability for neural networks with time-varying delay, Neurocomputing 101 (2013) $361-369$.

[31] T. Li, X. Ye, Improved stability criteria of neural networks with timevarying delays: An augmented LKF approach, Neurocomputing 73 (4-6) (2010) 1038 - 1047.

[32] D. Yue, E. Tian, Y. Zhang, A piecewise analysis method to stability analysis of linear continuous/discrete systems with time-varying delay, International Journal of Robust and Nonlinear Control 19 (13) (2009) 14931518

[33] Z. Yan, W. Zhang, G. Zhang, Finite-time stability and stabilization of itô stochastic systems with Markovian switching: mode-dependent parameter approach, IEEE Transactions on Automatic Control 60 (9) (2015) 2428-2433.

[34] W. Qi, X. Gao, J. Wang, Finite-time passivity and passification for stochastic time-delayed Markovian switching systems with partly known transition rates, Circuits, Systems, and Signal Processing 35 (11) (2016) 3913-3934.

[35] S. Onori, C. T. Abdallah, S. Galeani, P. Dorato, Finite time stability design via feedback linearization, in: 44th IEEE Conference on Decision and Control and The European Control Conference, Seville, Spain, 2005, pp. 4915-4920.

[36] G. Kamenkov, On stability of motion over a finite interval of time, Journal of Applied Mathematics and Mechanics 17 (2) (1953) 529-540.

[37] F. Amato, M. Ariola, P. Dorato, Finite-time control of linear systems subject to parametric uncertainties and disturbances, Automatica 37 (9) (2001) 1459-1463.

[38] Y. Ma, M. Chen, Finite time non-fragile dissipative control for uncertain TS fuzzy system with time-varying delay, Neurocomputing 177 (2016) $509-514$.

[39] J. Tan, C. Li, Finite-time stability of neural networks with impulse effects and time-varying delay, Neural Processing Letters (2016) 1-11.

[40] H. Zhao, L. Li, H. Peng, J. Xiao, Y. Yang, Finite-time boundedness analysis of memristive neural network with time-varying delay, Neural Processing Letters 44 (3) (2016) 665-679.

[41] C. Zhou, W. Zhang, X. Yang, C. Xu, J. Feng, Finite-time synchronization of complex-valued neural networks with mixed delays and uncertain perturbations, Neural Processing Letters (2017) 1-21.

[42] M. S. Ali, S. Saravanan, Robust finite-time $H_{\infty}$ control for a class of uncertain switched neural networks of neutral-type with distributed time varying delays, Neurocomputing 177 (2016) 454-468.

[43] S. He, F. Liu, Finite-time boundedness of uncertain time-delayed neural network with Markovian jumping parameters, Neurocomputing 103 (2013) 87-92

[44] D. Jiang, Finite time stability of Cohen-Grossberg neural network with time-varying delays, in: Advances in Neural Networks, Springer, 2009, pp. 522-531.

[45] X. Lin, H. Du, S. Li, Finite-time boundedness and $L^{2}$-gain analysis for switched delay systems with norm-bounded disturbance, Applied Mathematics and Computation 217 (12) (2011) 5982-5993.

[46] Y. Shen, C. Li, LMI-based finite-time boundedness analysis of neural networks with parametric uncertainties, Neurocomputing 71 (4) (2008) 502 507.

[47] S. Wang, T. Shi, L. Zhang, A. Jasra, M. Zeng, Extended finite-time $H_{\infty}$ control for uncertain switched linear neutral systems with time-varying delays, Neurocomputing 152 (2015) 377-387.
[48] Y. Wu, J. Cao, A. Alofi, A.-M. Abdullah, A. Elaiw, Finite-time boundedness and stabilization of uncertain switched neural networks with timevarying delay, Neural Networks 69 (2015) 135-143.

[49] Y. Zhang, P. Shi, S. K. Nguang, J. Zhang, H. R. Karimi, Finite-time boundedness for uncertain discrete neural networks with time-delays and Markovian jumps, Neurocomputing 140 (2014) 1-7.

[50] J. J. Hopfield, Neural networks and physical systems with emergent collective computational abilities, Proceedings of The National Academy of Sciences 79 (8) (1982) 2554-2558.

[51] Y. Kamp, M. Hasler, Recursive neural networks for associative memory, John Wiley \& Sons, Inc., 1990.

[52] C. Zheng, N. Li, J. Cao, Matrix measure based stability criteria for high-order neural networks with proportional delay, Neurocomputing 149 (2015) 1149-1154.

[53] J. K. Hale, S. M. V. Lunel, Introduction to functional differential equations, Vol. 99 of Applied Mathematical Sciences, Springer, 2013.

[54] Y. Li, L. Yang, Almost automorphic solution for neutral type high-order Hopfield neural networks with delays in leakage terms on time scales, Applied Mathematics and Computation 242 (2014) 679-693.

[55] A. Wu, Z. Zeng, Lagrange stability of memristive neural networks with discrete and distributed delays, IEEE transactions on Neural Networks and Learning Systems 25 (4) (2014) 690-703.

[56] J. Cao, J. Liang, J. Lam, Exponential stability of high-order bidirectional associative memory neural networks with time delays, Physica D: Nonlinear Phenomena 199 (34) (2004) 425 - 436.

[57] Dynamics of high-order Hopfield neural networks with time delays, Neurocomputing 73 (46) (2010) $820-826$

[58] F. Wang, D. Sun, H. Wu, Global exponential stability and periodic solutions of high-order bidirectional associative memory (BAM) neural networks with time delays and impulses, Neurocomputing 155 (2015) 261 276.

[59] J. Bai, R. Lu, A. Xue, Q. She, Z. Shi, Finite-time stability analysis of discrete-time fuzzy Hopfield neural network, Neurocomputing 159 (2015) 263-267.

[60] A. Berman, R. J. Plemmons, Nonnegative matrices in the mathematical sciences, Vol. 9 of Classics in Applied Mathematics, SIAM, 1994.

[61] Y. J. Cho, Y.-Q. Chen, Topological degree theory and applications, CRC Press, 2006

[62] X.-Y. Lou, B.-T. Cui, Novel global stability criteria for high-order Hopfield-type neural networks with time-varying delays, Journal of Mathematical Analysis and Applications 330 (1) (2007) 144-158.

[63] M. S. Ali, S. Arik, R. Saravanakumar, Delay-dependent stability criteria of uncertain Markovian jump neural networks with discrete interval and distributed time-varying delays, Neurocomputing 158 (2015) 167-173.

[64] S. Wang, T. Shi, M. Zeng, L. Zhang, F. E. Alsaadi, T. Hayat, New results on robust finite-time boundedness of uncertain switched neural networks with time-varying delays, Neurocomputing 151 (2015) 522-530.

[65] S. P. Boyd, L. El Ghaoui, E. Feron, V. Balakrishnan, Linear matrix inequalities in system and control theory, Vol. 15 of Studies in Applied and Numerical Mathematics, SIAM, 1994.

[66] B. Cooper, Stability analysis of higher-order neural networks for combinatorial optimization, International Journal of Neural Systems $12(03 \mathrm{n} 04)$ (2002) 177-186

[67] F. Mazenc, M. Malisoff, S.-I. Niculescu, Stability analysis for systems with time-varying delay: trajectory based approach, in: 54th IEEE Conference on Decision and Control, 2015, pp. 1811-1816.

[68] B. Zhou, A. V. Egorov, Razumikhin and Krasovskii stability theorems for time-varying time-delay systems, Automatica 71 (2016) 281-291.

[69] V. Singh, Simplified LMI condition for global asymptotic stability of delayed neural networks, Chaos, Solitons \& Fractals 29 (2) (2006) 470-473.

[70] J. Cheng, H. Zhu, Y. Ding, S. Zhong, Q. Zhong, Stochastic finite-time boundedness for Markovian jumping neural networks with time-varying delays, Applied Mathematics and Computation 242 (2014) 281-295.

[71] S. Rajavel, R. Samidurai, J. Cao, A. Alsaedi, B. Ahmad, Finite-time nonfragile passivity control for neural networks with time-varying delay, Applied Mathematics and Computation 297 (2017) 145-158.

[72] J. Lofberg, Yalmip : a toolbox for modeling and optimization in matlab, in: IEEE International Symposium on Computer Aided Control Systems Design, 2004, pp. 284-289.

[73] A. Arbi, C. Aouiti, F. Chérif, A. Touati, A. M. Alimi, Stability analysis of delayed Hopfield neural networks with impulses via inequality tech- 
niques, Neurocomputing 158 (2015) 281-294.

[74] R. Sakthivel, P. Vadivel, K. Mathiyalagan, A. Arunkumar, M. Sivachitra, Design of state estimator for bidirectional associative memory neural networks with leakage delays, Information Sciences 296 (2015) 263-274.

[75] F. Wang, M. Liu, Global exponential stability of high-order bidirectional associative memory (BAM) neural networks with time delays in leakage terms, Neurocomputing 177 (2016) 515-528. 